\title{
Material responses at micro- and macro-scales
}

\author{
M. Grigoriu \\ Cornell University, Ithaca NY 14853-3501, USA \\ Phone: 607-255-3334, e-mail: mdg12@cornell.edu
}

\begin{abstract}
Material properties at micro- and macro-scales used in micromechanics and continuum mechanics are random and deterministic and so are the corresponding material responses. We view material properties and responses in continuum mechanics as approximations of those in micromechanics. Our premise is that solutions of problems using material properties at various scales must agree in some sense, e.g., continuum mechanics solutions should match on average micromechanics solutions. Continuum solutions with this property are said to be consistent. Theoretical arguments and numerical examples are presented to demonstrate that the continuum solutions may or may not be consistent and may miss essential features of material response depending on the problem and quantity of interest. The examples include beams with random stiffness and one- and two-dimensional specimens with random conductivity.
\end{abstract}

Keywords: Apparent/effective properties, continuum mechanics, micromechanics, random field microstructure models, stochastic equations, stochastic mechanics

\section{Introduction}

At small scale, material properties fluctuate randomly in space and can be characterized by random functions calibrated to microstructure images. An example is a Markov random field calibrated to measurements of atomic lattice orientations in aluminum polycrystals [6]. The representative volume element (RVE) and the assumption that the microstructure random field is ergodic provide the link between microscale and continuum descriptions. The size of the RVE is, theoretically, infinite relative to the scale of fluctuation of microstructure properties which means for a polycrystal specimen that its size is much larger than that of the constitutive grains. Material properties at the RVE scale, referred to as bulk, macroscopic, global, or effective properties, are deterministic, do not depend on boundary conditions, and are used to define the constitutive relations at the macroscale, i.e., the scale of continuum mechanics [16] (Chap. 7). Material properties on finite specimens that are large relative to their constituents, referred to as apparent, are random and depend on boundary conditions. These specimens are said to be of the scale of the statistical volume element (SVE).

There are numerous studies on the upscaling of microstructure properties to apparent and effective material properties $[2,10,12,13,14,15]$. They show that averages of apparent 
properties can be used to develop bounds on effective properties [12, 15, 13]. For linear elasticity problems, these bounds are in the sense of quadratic forms so that they can only be used to bound global material responses by, e.g., energy norms of the type considered in [17]. To reduce calculations and capture material properties at small scale, it has been proposed to use apparent, rather than effective, material properties for response analysis. The implementation of this approach for linear elastic problems requires to select the SVE size for calculating apparent properties, calculate bounds on effective material properties corresponding to essential and natural boundary conditions, and solve two finite element problems for these bounds. The approach had mixed success because two opposite requirements: small finite elements for numerical accuracy and large finite elements such that the bounds on effective properties based on SVEs of finite element size are not too wide. An extensive study on this matter can be found in [17].

Our work relates closely to existing studies on the dependence of material responses on the resolution used to represent microstructure features. However, we propose a new framework for quantifying differences between material responses at different scales, the framework provided by the theory of stochastic differential equations. In this framework, material responses at different scales are solutions of stochastic/deterministic equations with the same functional form but different coefficients, which depend on the resolution used to represent material properties. The approach is beneficial since the theory of stochastic differential equations provides bounds on differences between solutions of equations with the same functional form but different coefficients, i.e., the case of material responses at different scales. The bounds are obtained from differences between the coefficients of these equations; they are not based on bounds on effective material properties derived from apparent material properties, a common approach in the literature. Moreover, the bounds can be constructed on various response metrics so that they can capture differences between local response features, a useful feature since these responses are sensitive to microstructure features [5]. Also, recent advances on practical methods for solving stochastic equations, see, e.g., [8] and [7] (Chaps. 8 and 9), provide efficient methods for solving complex stochastic problems of the type encountered in micromechanics.

Let $U(x)$ and $u_{0}(x), x \in D$, be micro- and macro-scale material response fields, where $D$ denotes the domain occupied by a material specimen. We assume throughout the paper that the probability law of the random field characterizing the microscale material properties is known and that $U(x)$ is the actual response. The microscale properties are used to infer effective material properties. The continuum mechanics solution $u_{0}(x)$ is viewed as an approximation of the microscale solution $U(x)$. If body forces and end conditions are deterministic, the micro- and macro-scale solutions are random and deterministic functions, so that it is only possible to compare $u_{0}(x)$, a deterministic function, with statistics of $U(x)$, a random function.

Our premise is that material responses based on material properties at different scales must agree in some sense, e.g., continuum mechanics solutions should match on average micromechanics solutions, i.e., $u_{0}(x)=E[U(x)], x \in D$, where $E[\cdot]$ denotes the expectation operator. Theoretical arguments and numerical examples are presented to evaluate the performance of the continuum solution $u_{0}(x)$ as an approximation of the microscale solution $U(x)$. The numerical examples include beams with random stiffness and one- and two-dimensional specimens with random conductivity, and are used to examine the consis- 
tency of the continuum solutions and quantify differences between continuum and microscale solutions. We focus on the lack of sensitivity of the continuum solution to some features of the material properties at small scale that affect significantly the microscale solution, e.g., the spatial correlation of the random field models for material properties at small scale, the difference and relationship between apparent and effective properties, and the inability of continuum solution to capture some relevant features of material response.

The following section presents two simple examples illustrating that the characterization of some quantities of interest require high resolution material models rather than bulk properties. Section 3 is somewhat technical. The first first part of this section develops bounds on the discrepancy between material responses corresponding to material models with various resolutions and apply them to bound differences between $u_{0}(x)$ and $U(x)$. The second part of this section constructs approximate solutions $U_{\text {perb }}(x)$ for the special case in which microstructure properties are small random perturbations about effective material properties and shows that $U_{\text {perb }}(x)$ is superior to $u_{0}(x)$. Section 4 further explores the relationships between $u_{0}(x)$ and $U(x)$ in the context of two examples, a beam with random stiffness and one- and two-dimensional specimens with random conductivity. Section 5 summarizes our findings on features and limitations of the continuum solution as an approximation of the microscale solution. Concluding remarks are presented in Sect. 6.

\section{Why microscale solutions}

Let $\mathcal{D}_{0}$ and $\mathcal{D}$ denote the operators defining the equations for $u_{0}(x)$ and $U(x)$. Since $\mathcal{D}$ is a random operator, the microscale solution is a random field whose probability law is defined by this operator, source terms, and boundary conditions. Generally, it is not possible to derive analytically the probability law of $U(x)$. Statistics of $U(x)$ can be inferred from samples of this random field obtained numerically from samples of the random entries of $\mathcal{D}$ by using existing deterministic solvers. The continuum mechanics solution $u_{0}(x)$ is a deterministic function that satisfies an equation defined by $\mathcal{D}_{0}$. The operators $\mathcal{D}$ and $\mathcal{D}_{0}$ have the same functional form but different coefficients, which reflect material properties at small and large scales.

The following two examples show that continuum solutions may or may not be consistent depending on the quantities of interest. The first example discusses a rod with random stiffness in tension. The second examines a parallel systems with random fibers.

Example 1. Consider a rod with length $l>0$ and random stiffness $A(x), 0 \leq x \leq l$, that is stretched at its ends by unit forces. The rod elongations $U(x)$ and $u_{0}(x), 0 \leq$ $x \leq l$, satisfy the differential equations $\mathcal{D}[U(x)]:=A(x) d U(x) / d x=1$ and $\mathcal{D}_{0}\left[u_{0}(x)\right]:=$ $A_{\text {eff }} d u_{0}(x) / d x=1$ with $U(0)=u_{0}(0)=0$, and have the expressions $U(x)=\int_{0}^{x} d y / A(y)=$ $\int_{0}^{x} B(y) d y$ and $u_{0}(x)=x / A_{\text {eff }}, 0<x<l$, where $B(x)=1 / A(x)$ and $A_{\text {eff }}$ denotes the effective stiffness. Suppose $B(x)$ is a homogeneous random field and let $\xi_{c}>0$ denote its correlation distance. If $\xi_{c} \gg l$, the samples of $B(x)$ are nearly invariant along the rod so that $U(x) \simeq x B_{0}=x / A_{0}$, where $B_{0}$ and $A_{0}$ denote random variables whose distributions are the marginal distributions of $B(x)$ and $A(x)$, respectively. The other limit, i.e., $\xi_{c} \ll l$, corresponds to the case in which the stiffness scale of fluctuation is much smaller that the rod length. If $B(x)$ is also ergodic, then $U(x) \simeq x E[B(\cdot)]$ for a sufficiently large $x$ and 
resembles the functional form of the continuum solution $u_{0}(x)=x / A_{\text {eff }}$. The continuum solution is consistent since it matches the expectation of the microscale solution, i.e., $u_{0}(x)=$ $E[U(x)]=\int_{0}^{x} E[1 / A(y)] d y=x E[1 / A(\cdot)]$, provided $1 / A_{\text {eff }}=E[1 / A(\cdot)]=E[B(\cdot)]$. The latter expectation coincides with the Reuss average, i.e., the limit of the spatial average $(1 / l) \int_{0}^{l} B(x) d x$ as $l \rightarrow \infty$ provided the compliance field is ergodic.

The solid heavy lines and the thin dash lines in Fig. 1 show the continuum mechanics
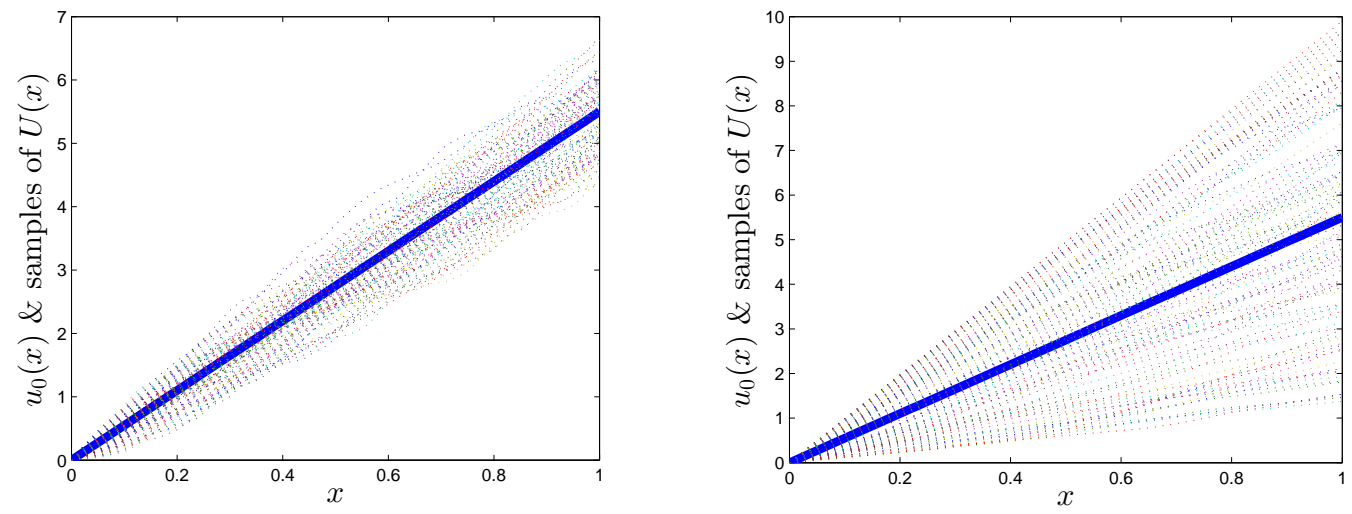

Figure 1: Continuum mechanics solution $u_{0}(x)$ and 50 samples of microscale solution $U(x)$ for $\rho=0.7$ (left panel) and $\rho=0.99$ (right panel)

solution $u_{0}(x)$ and 50 samples of the microscale solution $U(x)$ for the homogeneous translation field $B(x)=a+(b-a) \Phi(G(x)), 0 \leq x \leq l, l=1$, where $G(x)$ is homogeneous Gaussian field with mean 0 and variance 1 . Samples of $G(x)$ have been generated at discrete spatial coordinates $x_{i}=i \Delta x, \Delta x>0$, by the recurrence formula $G_{i}=\rho G_{i-1}+\sqrt{1-\rho^{2}} W_{i}, i \geq 1$, where $G_{i}=G\left(x_{i}\right),\left\{W_{i}\right\}$ are independent standard Gaussian variables $N(0,1),|\rho|<1$, and $G_{0} \sim N(0,1)$ is independent of $\left\{W_{i}\right\}$. The left and right panels in the figure are for $\rho=0.7$ and $\rho=0.99$. The samples of $U(x)$ resemble the continuum mechanics solution $u_{0}(x)$ for $\rho=0.99$ in agreement with our previous comments. However, their slopes are random following approximately the distribution of $B(0)$, rather than deterministic and equal to $1 / A_{\text {eff }}$. The plots show that the continuum mechanics solutions is consistent, e.g., $u_{0}(l)=5.50$ and estimates of $E[U(l)]$ based on 1000 samples of $U(x)$ are 5.5045 for $\rho=0.7$ and 5.6220 for $\rho=0.99$. However, the solutions $U(x)$ and $u_{0}(x)$ can differ significant, e.g., the standard deviations of $U(l)$, which coincides with the square root of the error $E\left[\left(U(l)-u_{0}(l)\right)^{2}\right]$, are 0.5795 for $\rho=0.7$ and $2.1279 \rho=0.99$. The distribution of the tip displacement $U(l)$ depends strongly on the relationship between the scale of fluctuation of the compliance field $B(x)$, which is controled by $\rho$ and the rod length $l$. This random variable is approximately Gaussian for $\rho \leq 0.5$ and non-Gaussian for strong correlations, e.g., estimates of the skewness and kurtosis coefficients of $U(l)$ based on 1000 independent samples are -0.1844 and 3.0114 for $\rho=0.5$ and -0.0703 and 2.0689 for $\rho=0.99$.

These results show that the continuum mechanics solution with $A_{\text {eff }}=1 / E[1 / A(x)]$ is consistent. However, it provides no information on the variability of $U(x)$ about $u_{0}(x)$, which depends in a complex manner on the spatial correlation of the stiffness random field. The continuum solution cannot be used to, e.g., design rods with imposed limits on their 
elongations.

Example 2. Consider a system consisting of $n$ fibers that experience the same elongation under loading and do not interact to each other. The system, referred to as parallel system, resembles a cable with parallel strings that are not in contact to each other. It is assumed that the system fibers/components are linearly elastic. Their stiffnesses $\left\{K_{i}\right\}$ are independent identically distributed random variables with distribution $F$, mean $\mu$, and variance $\sigma^{2}$. Under an imposed displacement $\delta>0$, the forces in individual components are $Q_{i}(\delta)=K_{i} \delta, i=$ $1, \ldots, n$, so that the total system response and the average force per component are $Q(\delta)=$ $\sum_{i=1}^{n} Q_{i}(\delta)=\left(\sum_{i=1}^{n} K_{i}\right) \delta$ and $Q_{\text {app }}(\delta)=\left(\sum_{i=1}^{n} K_{i} / n\right) \delta$. The relationships $Q_{i}(\delta)=K_{i} \delta$ and $Q_{\text {app }}(\delta)=K_{\text {app }} \delta$ define the input-output operators at small and large scale, respectively, where the system apparent stiffness $K_{\text {app }}=\sum_{i=1}^{n} K_{i} / n$ is random with mean $\mu=E\left[K_{\text {app }}\right]=$ $E\left[K_{1}\right]$ and variance $\sum_{i=1}^{n} \operatorname{Var}\left[R_{i}\right] / n^{2}=\operatorname{Var}\left[R_{1}\right] / n=\sigma^{2} / n$. This random variable converges almost surely to $\mu=E\left[K_{1}\right]$ by the strong law of large numbers ([19], Section 7.4 and 7.5), i.e., $K_{\text {app }} \rightarrow K_{\text {eff }}=E\left[K_{1}\right]$ as $n \rightarrow \infty$ under the stated conditions, where $K_{\text {eff }}$ denotes the effective stiffness.

The apparent and extreme forces in the system components are $Q_{\text {app }}(\delta)=K_{\text {app }} \delta$ and $Q_{\text {max }}(\delta)=\max _{1 \leq i \leq n}\left\{Q_{i}(\delta)\right\}=\max _{1 \leq i \leq n}\left\{K_{i}\right\} \delta$. For large systems $Q_{\text {app }}(\delta)$ approximates the macroscale solution $q_{0}(\delta)=\lim _{n \rightarrow \infty} Q_{\text {app }}(\delta)=E\left[K_{1}\right] \delta$, a deterministic solution that provides only a low resolution characterization for the internal forces in the system. The continuum solution is consistent since $q_{0}(\delta)=E\left[Q_{i}(\delta)\right]=E\left[K_{1}\right] \delta$.

The left and right panels in Fig. 2 show histograms of apparent and maximum forces
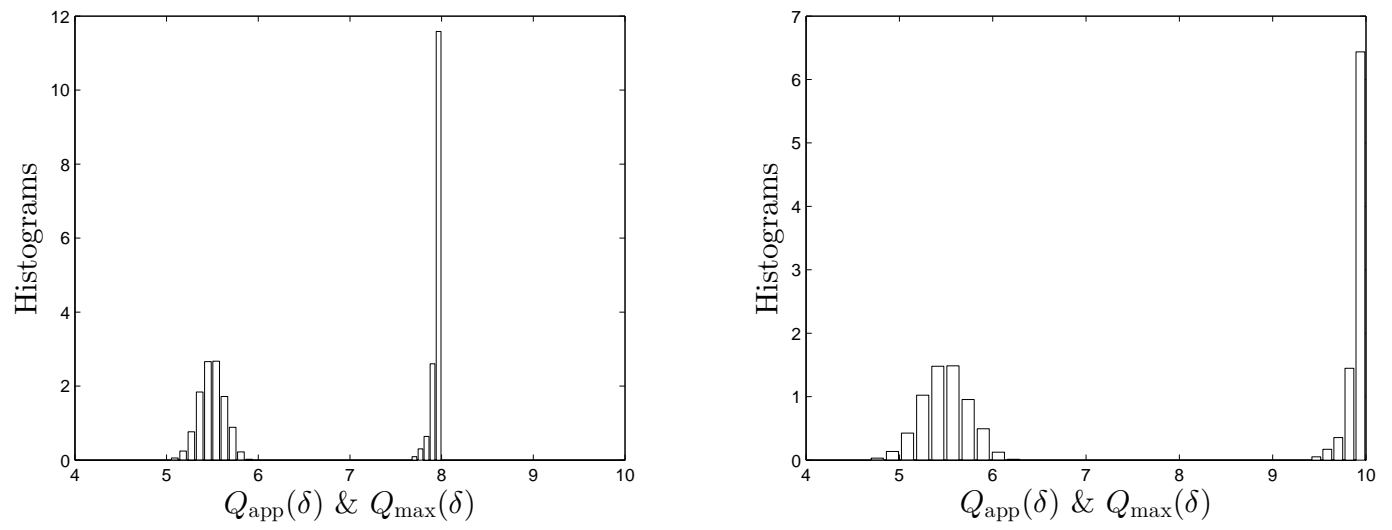

Figure 2: Histograms of $Q_{\text {app }}(\delta)$ and $Q_{\max }(\delta)$ for $\delta=1$ and $n=100$, based on 1000 independent samples for $K_{1} \sim U(a, b)$ with $(a, b)=(3,8)$ (left panel) and $(a, b)=(1,10)$ (right panel)

$Q_{\text {app }}(\delta)$ and $Q_{\max }(\delta)$ for $\delta=1, n=100$, and $K_{1} \sim U(a, b)$ with $(a, b)=(3,8)$ (left panel) and $(a, b)=(1,10)$ (right panel). For these properties of the random stiffness $K_{1}$, the apparent system forces have the same expectations, as illustrated by the left histograms of $Q_{\text {app }}(\delta)$ in both panels which are centered on the expectation $E\left[Q_{\text {app }}(\delta)\right]=(a+b) / 2=5.5$ of this random variable. On the other hand, the expectation of the largest component force $E\left[Q_{\max }(\delta)\right]$ depends on the uncertainty in $K_{1}$; it is 7.9526 for $(a, b)=(3,8)$ and 9.9147 for 
$(a, b)=(1,10)$. The continuum mechanics solutions $q_{0}(\delta)$ differs from $E\left[Q_{\max }(\delta)\right]$ so that it is not consistent for this quantity of interest.

In summary, the macroscale solution which approximate the random stiffnesses $\left\{K_{i}\right\}$ by either the apparent stiffness $K_{\text {app }}$ or the effective stiffness $K_{\text {eff }}$ are consistent since $q_{0}(\delta)=$ $E\left[Q_{\text {app }}(\delta)\right]=E\left[Q_{i}(\delta)\right]=E\left[K_{1}\right] \delta$. However, it is not consistent for the quantity of interest $Q_{\max }(\delta)$ since it differs from $E\left[Q_{\max }(\delta)\right]$. The latter quantity of interest, i.e., the extreme component force $Q_{\max }(\delta)$, is of particular interest in design.

\section{Material responses at different scales}

Let $u_{0}(x)$ and $U(x), x \in D$, denote material responses at large and small scales, i.e., the continuum mechanics and the microscale solutions, where $D$ is a bounded subset of $\mathbb{R}^{d}$, $d \leq 3$, occupied by a material specimen. These responses are solutions of the deterministic and stochastic equations

$$
\begin{aligned}
\mathcal{D}_{0}\left[u_{0}(x)\right] & =h(x), \quad x \in D, \\
\mathcal{D}[U(x)] & =h(x), \quad x \in D,
\end{aligned}
$$

where $\mathcal{D}_{0}$ and $\mathcal{D}$ are deterministic and stochastic differential operators that have the same functional but deterministic and random coefficients. We assume that these equations have the same deterministic source term $h(x)$ and deterministic boundary conditions so that differences between $u_{0}(x)$ and $U(x)$ are solely caused by the resolution of material models.

As previously stated, the microscale operator $\mathcal{D}$ and solution $U(x)$ are viewed as truth. The continuum mechanics operator $\mathcal{D}_{0}$ and solution $u_{0}(x)$ are regarded as approximations of the microscale operator and solution. Our objective is to examine similarities and differences between the continuum and microscale solutions. Since these solutions differ qualitatively, i.e., $u_{0}(x)$ is a deterministic function while $U(x)$ is random field, it is only possible to compare $u_{0}(x)$ with statistics of $U(x)$.

Consider for illustration the microscale solution $U(x)$ of the transport equation

$$
\mathcal{D}[U(x)]:=\nabla \cdot(A(x) \nabla U(x))=0, \quad x \in D=\left(0, l_{1}\right) \times\left(0, l_{2}\right) \subset \mathbb{R}^{2},
$$

with the boundary conditions $U\left(0, x_{2}\right)=0, U\left(l_{1}, x_{2}\right)=1$, and $\partial U(x) / \partial x_{2}=0$ for $x_{2}=0 ; l_{2}$, where $A(x)$ denotes the conductivity field at small scale that is assumed to be random. Since $A(x)$ is random so is the microscale solution $U(x)$. The macroscale solution $u_{0}(x)$ satisfies Eq. 2 with $A_{\text {eff }}$ in place of $A(x)$, i.e.,

$$
\mathcal{D}_{0}\left[u_{0}(x)\right]:=\nabla \cdot\left(A_{\mathrm{eff}} \nabla u_{0}(x)\right)=0, \quad x \in D=\left(0, l_{1}\right) \times\left(0, l_{2}\right) \subset \mathbb{R}^{2},
$$

and has the expression $u_{0}(x)=x_{1} / l_{1}, x=\left(x_{1}, x_{2}\right) \in D$.

Our objectives are to assess differences and similarities between the micro- and macroscale solutions $U(x)$ and $u_{0}(x)$ based on theoretical arguments and numerical illustrations. Since $U(x)$ and $u_{0}(x)$ satisfy differential equations with the same functional form and different coefficients, the theory of stochastic differential equations can be used to examine the discrepancy between these solution. This observation allows us to construct a broad range 
of metrics for the discrepancy between $U(x)$ and $u_{0}(x)$ rather than just global metrics of the type available in the literature, e.g., energy norms [17].

The following two subsections (1) develop bounds on the discrepancy between solutions corresponding to material models with various resolutions and apply these bounds to quantify differences between macroscale and microscale solutions and (2) construct approximate solutions for the special case in which microscale properties have relatively small uncertainty. The considerations in these and subsequent sections show that the proposed framework based on the theory of stochastic differential equations provides a useful tool for assessing differences between material responses at different scales.

\subsection{Discrepancy between solutions at different scales}

We calculate the discrepancies between the micro- and macro-scale solutions $U(x)$ and $u_{0}(x)$ for a real-valued conductivity random field $A(x)$ that is physically consistent, i.e., it is bounded and positive. Property 2 is the main result. It shows that the discrepancy between solutions of depends essentially on the difference between material properties at the scales under considerations.

Throughout this it is assumed that (1) the conductivity field $A(x), x \in D$, is a realvalued, homogeneous, non-Gaussian random field defined on a probability space $(\Omega, \mathcal{F}, P)$ whose samples reside in a bounded interval $[a, b], 0<a<b<\infty$, of the real line with probability 1 and $(2) A(x)$ is sufficiently smooth so that its partial derivatives $A(x)$ with respect to spatial coordinates are in $L^{2}(D \times \Omega)$, where $L^{2}(D \times \Omega)$ denotes the family of realvalues square integrable functions, i.e., functions $g: D \times \Omega \rightarrow \mathbb{R}$ such that $E\left[\int_{D} g(x)^{2} d x\right]=$ $\int_{D \times \Omega} g(x, \omega)^{2} d x P(d \omega)<\infty$.

We recast Eq. 2 in an equation whose solution vanishes on the Dirichlet boundaries $\partial D_{d}=\{0\} \times\left(0, l_{2}\right) \cup\left\{l_{1}\right\} \times\left(0, l_{2}\right)$ by the change of variables $V(x)=U(x)-x_{1} / l_{1}$. With the notation $C(x)=\left(1 / l_{1}\right) \partial A(x) / \partial x_{1}$, the random field $V(x)$ is defined by

$$
-\nabla \cdot(A(x) \nabla V(x))=C(x), \quad x \in D=\left(0, l_{1}\right) \times\left(0, l_{2}\right) \subset \mathbb{R}^{2},
$$

with the boundary conditions $V\left(0, x_{2}\right)=V\left(l_{1}, x_{2}\right)=0$, and $\partial V(x) / \partial x_{2}=0$ for $x_{2}=0 ; l_{2}$ since $\nabla U(x)=\nabla V(x)+\beta$, where $\beta$ is a two-dimensional column vector with entries $1 / l_{1}$ and 0 .

Consider the linear space

$\mathcal{W}(D, \Omega)=\left\{W: D \times \Omega \rightarrow \mathbb{R}, W \in L^{2}(D \times \Omega), \nabla W \in L^{2}(D \times \Omega), W(x)=0, x \in \partial D_{d}, P-\right.$ a.s. $\}$

and the bilinear function $\mathcal{B}: \mathcal{W}(D, \Omega) \times \mathcal{W}(D, \Omega) \rightarrow \mathbb{R}$ defined by

$$
\mathcal{B}(V, W)=E\left[\int_{D} A(x) \nabla V(x) \cdot \nabla W(x) d x\right] .
$$

It can be shown [18] (Sect. 9.2) that $\langle U, W\rangle_{\mathcal{W}(D, \Omega)}=E\left[\int_{D}(U(x) W(x)+\nabla U(x) \cdot \nabla W(x)) d x\right]$ is an inner product on $\mathcal{W}(D, \Omega)$ and that $\mathcal{W}(D, \Omega)$ is a Hilbert space with the norm $\|W\|_{\mathcal{W}(D, \Omega)}^{2}=\langle W, W\rangle_{\mathcal{W}(D, \Omega)}$. 
The weak form of Eq. 4 results by multiplying this equation with $W \in \mathcal{W}(D, \Omega)$ arbitrary, integrating the resulting equation over $D \times \Omega$, and using Green's theorem. Our objective is to find the weak solution of Eq. 4, i.e., find a unique $V \in \mathcal{W}(D, \Omega)$ such that

$$
\mathcal{B}(V, W)=\langle C, W\rangle_{L^{2}(D \times \Omega)}, \quad \forall W \in \mathcal{W}(D, \Omega) .
$$

where $\langle C, W\rangle_{L^{2}(D \times \Omega)}=E\left[\int_{D} C(x) W(x) d x\right]=\int_{D \times \Omega} C(x, \omega) W(x, \omega) d x P(d \omega)$ denotes the inner product in the space of square integrable functions $L^{2}(D \times \Omega)$.

Property 1. If $A, C: D \times \Omega \rightarrow \mathbb{R}$ are $\mathcal{G} \times \mathcal{F}$-measurable and $\lambda \times P$-integrable and $C \in L^{2}(D \times \Omega)$, then Eq. 7 admits a unique solution, where $\mathcal{G}$ denotes the Borel $\sigma$-field on $D$ and $\lambda$ is the Lebesgue measure on $\mathbb{R}^{d}$.

Proof: The Lax-Milgram theorem, see, e.g., [4] (Sect. 3.1.3) and [18] (Theorem 13, p. 166), and the facts that the bilinear form in Eq. 6 is such that there exist constants $\alpha, \beta>0$ such that $|\mathcal{B}(V, W)| \leq \alpha\|V\|_{\mathcal{W}(D, \Omega)}\|W\|_{\mathcal{W}(D, \Omega)}$ and $\mathcal{B}(V, V) \geq \beta\|V\|_{\mathcal{W}(D, \Omega)}^{2}$ [18] (Sect. 7.5, Theorem 9) and that the inner product $\langle C, W\rangle_{L^{2}(D \times \Omega)}$ is a linear continuous functional of $W$ yield the stated property, see, e.g., [7] (Sect. 9.4.3).

Consider a simpler version $\tilde{A}(x)$ of $A(x)$, e.g., a parametric random field with small stochastic dimension that is defined on the probability space $(\Omega, \mathcal{F}, P)$ and has the same properties as $A(x)$. Denote by $\tilde{V}(x)$ the solution of Eq. 4 with $\tilde{A}(x)$ in place of $A(x)$, i.e., the equation $-\nabla \cdot(\tilde{A}(x) \nabla V(x))=\tilde{C}(x)$ with the boundary conditions of Eq. 4, where $\tilde{C}(x)=\left(1 / l_{1}\right) \partial \tilde{A}(x) / \partial x_{1}$. The weak form of this equation is

$$
\begin{aligned}
\tilde{\mathcal{B}}(\tilde{V}, W) & =E\left[\int_{D} \tilde{A}(x) \nabla \tilde{V}(x) \cdot \nabla W(x) d x\right] \\
& =\langle\tilde{C}, W\rangle_{L^{2}(D \times \Omega)}=\int_{D \times \Omega} \tilde{C}(x, \omega) W(x, \omega) d x P(d \omega), \quad \forall W \in \mathcal{W}(D, \Omega) .
\end{aligned}
$$

Property 2. The discrepancy between the weak solutions of $V(x)$ and $\tilde{V}(x)$ of Eqs. 7 and 8 can be bounded by

$$
\|V-\tilde{V}\|_{\mathcal{W}(D, \Omega)} \leq \frac{1}{\beta}\left(\|A-\tilde{A}\|_{L^{\infty}(D \times \Omega)}\|\tilde{V}\|_{\mathcal{W}(D, \Omega)}+\|C-\tilde{C}\|_{L^{2}(D \times \Omega)}\right),
$$

where $\beta>0$ is constant.

Proof: We have

$$
\begin{aligned}
& |\mathcal{B}(V-\tilde{V}, W)| \leq|\mathcal{B}(V, W)-\mathcal{B}(\tilde{V}, W)+\tilde{\mathcal{B}}(\tilde{V}, W)-\tilde{\mathcal{B}}(\tilde{V}, W)| \\
& \leq|\tilde{\mathcal{B}}(\tilde{V}, W)-\mathcal{B}(\tilde{V}, W)|+|\mathcal{B}(V, W)-\tilde{\mathcal{B}}(\tilde{V}, W)| \\
& \quad=|\tilde{\mathcal{B}}(\tilde{V}, W)-\mathcal{B}(\tilde{V}, W)|+\left|\langle C-\tilde{C}, W\rangle_{L^{2}(D \times \Omega)}\right|
\end{aligned}
$$


by properties of $\mathcal{B}$ and Eqs. 7-8. The first term of the latter expression can be bounded by

$$
\begin{aligned}
|\tilde{\mathcal{B}}(\tilde{V}, W)-\mathcal{B}(\tilde{V}, W)| & =\left|E\left[\int_{D}(\tilde{A}(x)-A(x)) \nabla \tilde{V}(x) \cdot \nabla W(x) d x\right]\right| \\
& \leq\left(E\left[\int_{D}(A(x)-\tilde{A}(x))^{2} \nabla \tilde{V}(x) \cdot \nabla \tilde{V}(x)\right] E\left[\int_{D} \nabla W(x) \cdot \nabla W(x)\right]\right)^{1 / 2} \\
& \leq\|A-\tilde{A}\|_{L^{\infty}(D \times \Omega)}\|\tilde{V}\|_{\mathcal{W}(D, \Omega)}\|W\|_{\mathcal{W}(D, \Omega)}
\end{aligned}
$$

by the Cauchy-Schwarz inequality, the definition of the norm on $\mathcal{W}(D, \Omega)$, and the assumption that all samples of $A(x)$ and $\tilde{A}(x)$ are bounded in $D$ which implies $\|A-\tilde{A}\|_{L^{\infty}(D \times \Omega)}<$ $\infty$ almost surely, i.e., $\max _{x \in D}\{|A(x, \omega)-\tilde{A}(x, \omega)|\}$ is bounded for almost all samples of $A(x)-\tilde{A}(x)$ in $D$. The following upper bound, on the second term results

$$
\left|\langle C-\tilde{C}, W\rangle_{L^{2}(D \times \Omega)}\right| \leq\|C-\tilde{C}\|_{L^{2}(D \times \Omega)}\|W\|_{L^{2}(D \times \Omega)} \leq\|C-\tilde{C}\|_{L^{2}(D \times \Omega)}\|W\|_{\mathcal{W}(D, \Omega)},
$$

on the second term results by using the Cauchy-Schwarz inequality and the definition of the norm in $\mathcal{W}(D, \Omega)$. This gives

$$
|\mathcal{B}(V-\tilde{V}, W)| \leq\|A-\tilde{A}\|_{L^{\infty}(D \times \Omega)}\|\tilde{V}\|_{\mathcal{W}(D, \Omega)}\|W\|_{\mathcal{W}(D, \Omega)}+\|C-\tilde{C}\|_{L^{2}(D \times \Omega)}\|W\|_{\mathcal{W}(D, \Omega)}
$$

and

$|\mathcal{B}(V-\tilde{V}, V-\tilde{V})| \leq\|A-\tilde{A}\|_{L^{\infty}(D \times \Omega)}\|\tilde{V}\|_{\mathcal{W}(D, \Omega)}\|V-\tilde{V}\|_{\mathcal{W}(D, \Omega)}+\|C-\tilde{C}\|_{L^{2}(D \times \Omega)}\|V-\tilde{V}\|_{\mathcal{W}(D, \Omega)}$.

for $W=V-\tilde{V}$. The property $\mathcal{B}(U, U) \geq \beta\|U\|_{\mathcal{W}(D, \Omega)}^{2}, \beta>0$, implies

$\beta\|V-\tilde{V}\|_{\mathcal{W}(D, \Omega)}^{2} \leq\|A-\tilde{A}\|_{L^{\infty}(D \times \Omega)}\|\tilde{V}\|_{\mathcal{W}(D, \Omega)}\|V-\tilde{V}\|_{\mathcal{W}(D, \Omega)}+\|C-\tilde{C}\|_{L^{2}(D \times \Omega)}\|V-\tilde{V}\|_{\mathcal{W}(D, \Omega)}$,

which yields Eq. 9.

Continuum mechanics. Material responses at large scale are the solutions $u_{0}(x)=x_{1} / l_{1}$ of Eq. 3 or $v_{0}(x)=0$ of Eq. 4 with $A_{\text {eff }}$ in place of $A(x)$. The bound in Eq. 9 with $v_{0}(x)$ in place of $\tilde{V}(x)$ gives

$$
\left\|U-u_{0}\right\|_{\mathcal{W}(D, \Omega)}=\|V\|_{\mathcal{W}(D, \Omega)} \leq(1 / \beta)\|C\|_{L^{2}(D \times \Omega)} .
$$

The bound is controlled by the rate of change of the random conductivity field $A(x)$ with the spatial coordinate $x_{1}$. The bound becomes tighter as the rate of change of $A(x)$ with $x_{1}$ decreases.

Special case. Bounds similar to those in Eq. 9 can be obtained for strong solutions of some stochastic equations. For example, let $U(x)$ be the solution of the one-dimensional version $d\left(A(x) U^{\prime}(x)\right) / d x=0, x \in D=(0, l), l>0$, of Eq. 2 with the boundary conditions $U(0)=0$ and $U(l)=1$, where $A(x)$ has the same properties as $A(x)$ in this equation except it is defined on the real line rather than $\mathbb{R}^{2}$. The solution of this equation is $U(x)=I(x) / I(l)$, $0 \leq x \leq l$, where $I(x)=\int_{0}^{x} d y / A(y)$.

Let $\tilde{A}(x)$ be an approximate conductivity field $A(x)$ that has similar properties. The solution $\tilde{U}(x)$ of the above stochastic differential equation with $\tilde{A}(x)$ in place of $A(x)$ is 
$\tilde{U}(x)=\tilde{I}(x) / \tilde{I}(l), 0 \leq x \leq l$, where $\tilde{I}(x)=\int_{0}^{x} d y / \tilde{A}(y)$. The discrepancy between $U(x)$ and $\tilde{U}(x)$ can be bounded by

$$
\begin{aligned}
|U(x)-\tilde{U}(x)| & =\frac{|I(x) \tilde{I}(l)-\tilde{I}(x) I(l)|}{I(l) \tilde{I}(l)} \leq \frac{\tilde{I}(l)|I(x)-\tilde{I}(x)|+\tilde{I}(x)|\tilde{I}(l)-I(l)|}{I(l) \tilde{I}(l)} \\
& \leq \frac{\tilde{I}(l)\left(x / a^{2}\right)\|A-\tilde{A}\|_{L^{\infty}((0, x) \times \Omega)}+\tilde{I}(x)\left(l / a^{2}\right)\|A-\tilde{A}\|_{L^{\infty}(D \times \Omega)}}{I(l) \tilde{I}(l)} \\
& \leq\left(l / a^{2}\right) \frac{\tilde{I}(l)+\tilde{I}(x)}{I(l) \tilde{I}(l)}\|A-\tilde{A}\|_{L^{\infty}(D \times \Omega)} \leq \frac{2\left(l / a^{2}\right)}{I(l)}\|A-\tilde{A}\|_{L^{\infty}(D \times \Omega)},
\end{aligned}
$$

since $|I(x)-\tilde{I(x)}| \leq \int_{0}^{x} d y|\tilde{A}(y)-A(y)| /(A(y) \tilde{A}(y)) \leq\|A-\tilde{A}\|_{L^{\infty}((0, x) \times \Omega)} \int_{0}^{x} d y /(A(y) \tilde{A}(y))$ and $\|A-\tilde{A}\|_{L^{\infty}((0, x) \times \Omega)} \leq\|A-\tilde{A}\|_{L^{\infty}(D \times \Omega)}$ and $\int_{0}^{x} d y /(A(y) \tilde{A}(y)) \leq l / a^{2}$ for $x \in D$. The discrepancy between the exact and approximate solutions $U(x)$ and $\tilde{U}(x)$ can also be bounded by

$$
|U(x)-\tilde{U}(x)| \leq \frac{2\left(l / a^{2}\right)}{\tilde{I}(l)}\|A-\tilde{A}\|_{L^{\infty}(D \times \Omega)}, \quad x \in D,
$$

which results by interchanging the integrals $I(x)$ and $\tilde{I}(x)$ in the previous bound. The above bounds show that the approximate strong solution $\tilde{U}(x)$ converges uniformly, $P$-almost surely to $U(x)$ in $D$ as the discrepancy between the approximate and exact random fields vanishes, i.e., $\|A-\tilde{A}\|_{L^{\infty}(D \times \Omega)} \rightarrow 0$.

Suppose now that $\tilde{A}(x)$ is approximated by a deterministic function $\tilde{a}(x)$ with the same properties as the samples of $A(x)$. The discrepancy between the solution $\tilde{u}(x)$ corresponding to the conductivity filed $\tilde{a}(x)$ and $U(x)$ can be bounded by

$$
\begin{aligned}
& |U(x)-\tilde{u}(x)| \leq \frac{2\left(l / a^{2}\right)}{I(l)}\|A-\tilde{a}\|_{L^{\infty}(D \times \Omega)} \text { and } \\
& |U(x)-\tilde{u}(x)| \leq \frac{2\left(l / a^{2}\right)}{\tilde{I}(l)}\|A-\tilde{a}\|_{L^{\infty}(D \times \Omega)},
\end{aligned}
$$

where $\|A-\tilde{a}\|_{L^{\infty}(D \times \Omega)}=\max _{x \in D, \omega \in \Omega \backslash \Omega_{0}}|A(x, \omega)-\tilde{a}(x)|$ and $\Omega_{0}$ is a measurable subset of $\Omega$ with $P\left(\Omega_{0}\right)=0$. In contrast to the first bound which is random, the second bound is deterministic and shows that the discrepancy between $U(x)$ and $\tilde{u}(x)$ can be bounded uniformly $P$-almost surely in $D$.

The continuum mechanics solution corresponds to the case in which $\tilde{a}(x)=a_{0}$ is a constant, so that $\tilde{u}(x)=u_{0}(x)=x / l, x \in D=(0, l)$. Numerical results in Fig. 3 are for $l=1$ and a homogeneous translation field $A(x)$ with Beta marginal distribution of range $(a, b)=(1,10)$ and shape parameters $(p, q)=(1,3)$ (see Eq. 16 in Sect. 4.2 for details). The Gaussian image of $A(x)$ is a homogeneous random field with mean 0, variance 1, and exponential correlation with decay parameter $\lambda=20$. The left and middle panels of Fig. 3 shows 50 samples of $U(x)-\tilde{u}(x), x \in D=(0,1)$, and a histogram of $\max _{x \in D}\{|U(x)-\tilde{u}(x)|\}$ based on 1000 independent samples of this random variable. The right panel in the figure shows a histogram of the random flow $A(x) U^{\prime}(x)=1 / \int_{0}^{l} d y / A(y)$ through the specimen. The heavy dots give the range of the flow $A_{\text {eff }} u_{0}^{\prime}(x)=A_{\text {eff }} / l$ under the continuous mechanics model with $A_{\text {eff }}$ in the range provided by the Reuss and Voigt bounds. 

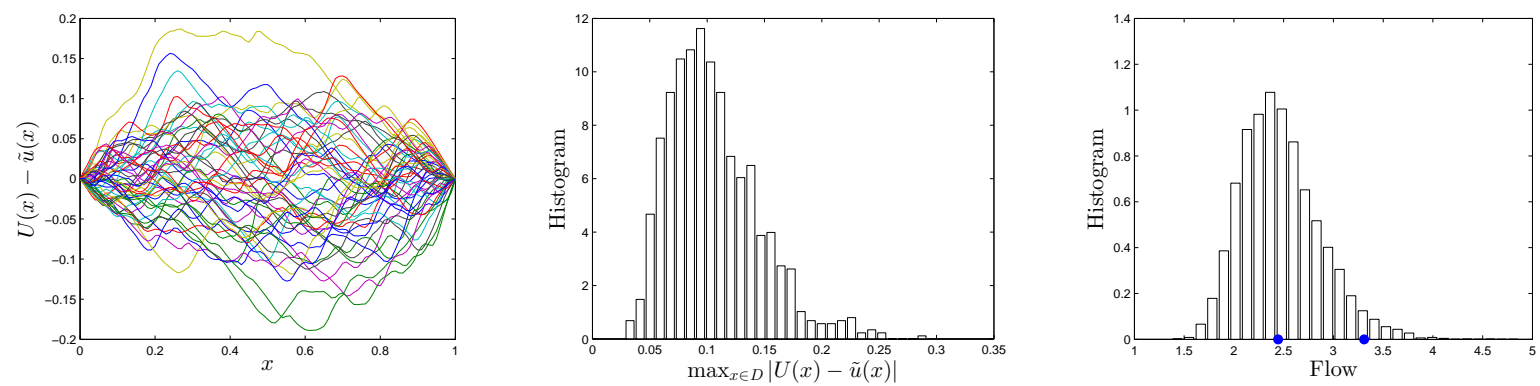

Figure 3: Samples of $U(x)-\tilde{u}(x)$ (left panel), histogram of $\max _{x \in D}\{|U(x)-\tilde{u}(x)|\}$ (middle panel), and flow histogram (right panel) for $\lambda=20$

The continuum mechanics solution $u_{0}(x)=\tilde{u}(x)=x$ and the flows through the specimen are consistent for $A_{\text {eff }}=1 / E[1 / A(x)]$, e.g., estimates of the expectation $E\left[A(x) U^{\prime}(x)\right]$ of the microscale flow and the Reuss bound $1 / E[1 / A(x)]$ based on 10000 samples are 2.4687 and 2.4439. However, there are significant differences between the microscale and continuum mechanics solutions. The continuum mechanics solution $u_{0}(x)=\tilde{u}(x)=x$ is deterministic and independent of material properties, while the microscale solution is random and depends strongly on material properties as illustrated by the samples $U(x)-\tilde{u}(x)$. The flow predicted by the continuum model is a number between the heavy dots in the right panel of Fig. 3, while the actual flow is a random variable with properties that depend on the conductivity field $A(x)$, the specimen size $l$, and boundary conditions. Estimates of the flow mean, standard deviation, skewness, and kurtosis based on 10000 flow samples are 2.4687, 0.3997, 0.7146, and 3.8763. These estimates become 1.9229, 0.8853, 1.2613, and 5.3953 for $A(x)$ in the range $(a, b)=(0.1,20)$ rather than $(a, b)=(1,10)$. The average flows given by the continuum solution provides very limited information on the actual flow, a non-Gaussian variable whose properties depend strongly on the probability law of the conductivity field, e.g., the flow kurtosis coefficients are 3.8763 for the range $(a, b)=(1,10)$ of the conductivity field and 5.3953 for $(a, b)=(0.1,20)$. These changes in the flow distribution cannot be captured by the continuum solution, a significant limitation if we design for flow.

\subsection{Small random fluctuations}

We consider the special case in which the random fluctuations of the conductivity field $A(x)$ are small with respect to a constant value $A_{0}$, i.e., the conductivity field $A(x)$ in Eq. 2 has the form $A(x)=A_{0}+\varepsilon \tilde{A}(x)$, where $\varepsilon>0$ is a small parameters and $A_{0}>0$ is a constant of order $1 \gg \varepsilon$, e.g., $A_{0}$ can be the effective conductivity. The random conductivity field $A(x)$ constitutes a small random perturbation about the effective conductivity.

We represent the solution of Eq. 4 by the perturbation series $V(x)=V_{0}(x)+\varepsilon V_{1}(x)+$ $\varepsilon^{2} V_{2}(x)+\cdots$, where the functions $V_{0}(x)=v_{0}(x)$ and $V_{1}(x)$ satisfy the differential equations

$$
\begin{aligned}
& -\Delta V_{0}(x)=0 \\
& -\Delta V_{1}(x)=\frac{1}{A_{0} l_{1}} \frac{\partial \tilde{A}(x)}{\partial x_{1}}
\end{aligned}
$$


with the boundary conditions $V_{k}\left(0, x_{2}\right)=V_{k}\left(l_{1}, x_{2}\right), x_{2} \in\left(0, l_{2}\right)$, and $\partial V_{k}\left(x_{1}, x_{2}\right) / \partial x_{2}=0$ for $x_{2}=0 ; l_{2}, x_{1} \in\left(0, l_{1}\right), k=0,1$. The first equation gives $V_{0}(x)=0, x \in D$. The right side of the second equation is stochastic so that $V_{1}(x)$ is a random field whose properties depend essentially on the rate of change of $\tilde{A}(x)$ along the $x_{1}$-coordinate quantified by the partial derivative $\partial \tilde{A}(x) / \partial x_{1}$. The constant conductivity $A_{0}$ only scales $V_{1}(x)$. The series representation of the solution $V(x)$ holds if $V_{1}(x) \mid \sim O(1)$ or smaller, a requirement that imposes constraints on $\partial \tilde{A}(x) / \partial x_{1}$.

The discrepancy between the continuum mechanics and microscale solutions the of continuum mechanics and microstructures can be bounded by Eq. 9, which gives

$$
\left\|V-V_{0}\right\|_{\mathcal{W}(D, \Omega)}=\left\|\varepsilon V_{1}(x)+\varepsilon^{2} V_{2}(x)+\cdots\right\|_{\mathcal{W}(D, \Omega)} \leq \frac{\varepsilon}{\rho l_{1}}\left(E\left[\int_{D}\left(\frac{\partial \tilde{A}(x)}{\partial x_{1}}\right)^{2} d x\right]\right)^{1 / 2}
$$

The same bound holds for the discrepancy $\left\|U-U_{0}\right\|_{\mathcal{W}(D, \Omega)}=\left\|U-u_{0}\right\|_{\mathcal{W}(D, \Omega)}$ between the corresponding solutions $U(x)=V(x)+x_{1} / l_{1}$ and $U_{0}(x)=u_{0}(x)=V_{0}(x)+x_{1} / l_{1}$ of Eq. 2.

Example 3. Let $U(x)$ be the solution of the one-dimensional version $d\left(A(x) U^{\prime}(x)\right) / d x=0$, $x \in D=(0, l), l>0$, of Eq. 2 with the boundary conditions $U(0)=0$ and $U(l)=1$. We continue to consider the conductivity model $A(x)=A_{0}+\varepsilon \tilde{A}(x)$. The members of the perturbation series $U(x)=U_{0}(x)+\varepsilon U_{1}(x)+\varepsilon^{2} U_{2}(x)+\cdots$ have the expressions

$$
\begin{aligned}
& U_{0}(x)=u_{0}(x)=x / l \text { and } \\
& U_{1}(x)=\frac{1}{A_{0} l}\left[\frac{x}{l} \int_{0}^{l} \tilde{A}(y) d y-\int_{0}^{x} \tilde{A}(y) d y\right]
\end{aligned}
$$

since $d\left(A(x) U^{\prime}(x)\right) / d x=0$ implies $U_{0}^{\prime \prime}(x)=0$ with the boundary conditions $U_{0}(0)=0$ and $U_{0}(l)=1$ and $U_{1}^{\prime \prime}(x)=-\tilde{A}^{\prime}(x) /\left(A_{0} l\right)$ with zero boundary conditions. The first order perturbation and the exact solutions have the expressions

$$
\begin{aligned}
U_{\text {pert }}(x) & =\frac{x}{l}+\varepsilon \frac{1}{A_{0} l}\left[\frac{x}{l} \int_{0}^{l} \tilde{A}(y) d y-\int_{0}^{l} \tilde{A}(y) d y\right] \text { and } \\
U(x) & =\frac{\int_{0}^{x} d y / A(y)}{\int_{0}^{l} d y / A(y)}
\end{aligned}
$$

The bound in Eq. 11 can be used to quantify the discrepancy between the exact and the perturbation solutions. This bound depends essentially on an expectation involving the spatial derivative of the conductivity field.

Following numerical results are for $A_{0}=1$ and $\tilde{A}(x)=\Phi(G(x)), x \in D=(0,1)$, where $G(x)$ is a homogeneous Gaussian field with mean 0, variance 1, and constant onesided spectral density in the frequency band $(0, \bar{\nu}), \bar{\nu}=50$. An estimate of the square root of the expectation $E\left[\int_{D}\left(\partial \tilde{A}(x) / \partial x_{1}\right)^{2} d x\right]$ in the bound of Eq. 11 is 8.78, which shows that fluctuations of order $\varepsilon$ in the conductivity field are mapped in errors of $U_{\text {pert }}(x)$ relative to $U(x)$ of the same order.

The solid and dash lines in the left and middle panels of Fig. 4 are samples of $U(x)$ and $U_{\text {pert }}(x)$ for $\varepsilon=0.5$ (left panel) and $\varepsilon=0.7$ (middle panel). The solid and dash lines in the 

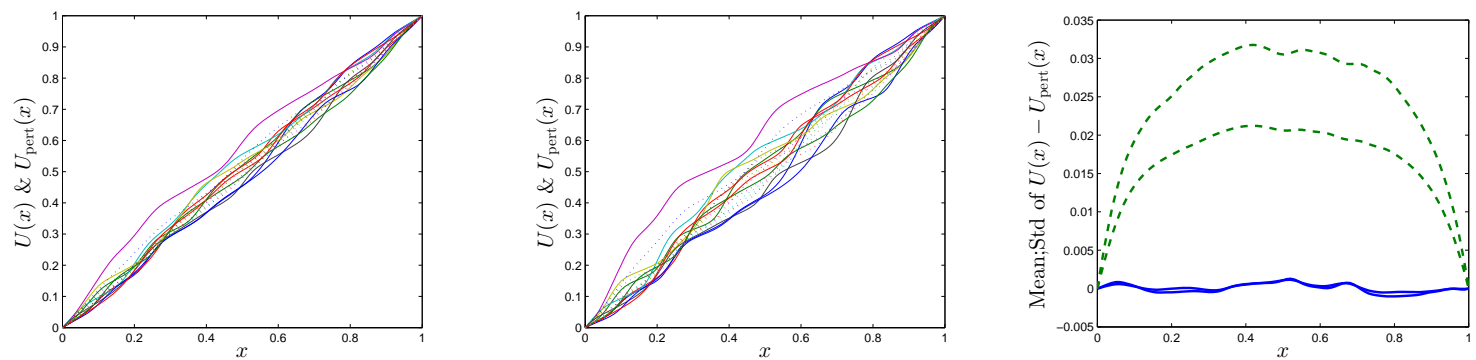

Figure 4: Ten samples of $U(x)$ (solid lines) and $U_{\text {pert }}(x)$ (dash lines) for $\varepsilon=0.5$ (left panel) and $\varepsilon=0.7$ (middle panel) and the means and standard deviations of $U(x)-U_{\text {pert }}(x)$ in solid and dash lines (right panel)
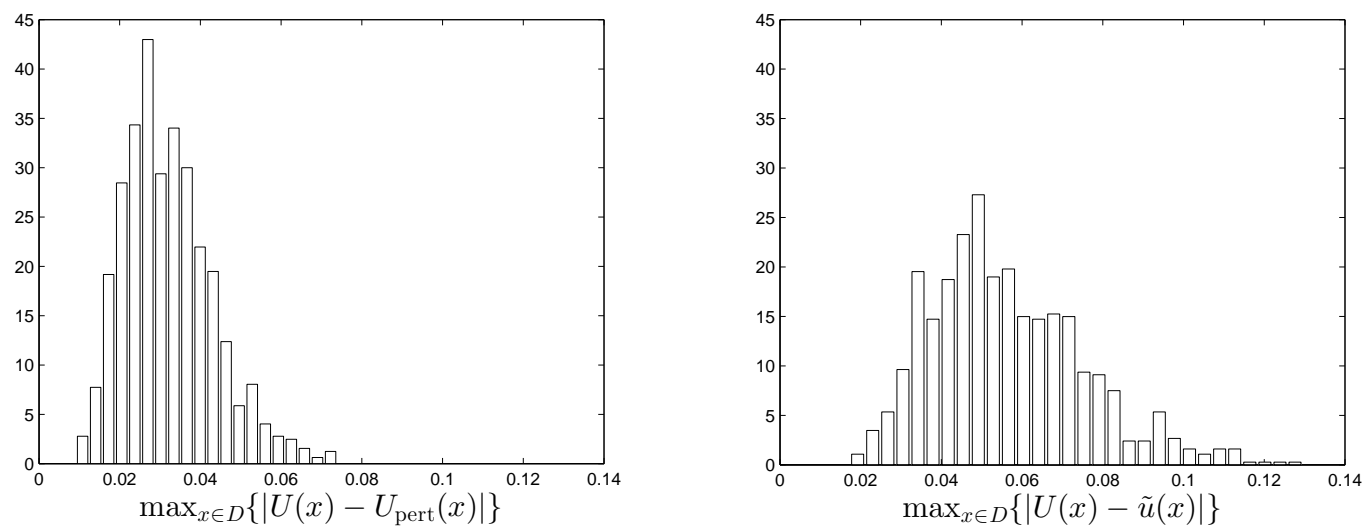

Figure 5: Histograms of $\max _{x \in D}\left\{\left|U(x)-U_{\text {pert }}(x)\right|\right\}$ (left panel) and $\max _{x \in D}\left\{\left|U(x)-u_{0}(x)\right|\right\}$ (right panel) for $\varepsilon=0.5$

right panel are the expectations and the standard deviations of $U(x)-U_{\text {pert }}(x)$ for $\varepsilon=0.5$ and $\varepsilon=0.7$; the larger standard deviation function is for $\varepsilon=0.7$. The exact and perturbation solutions have similar averages but their higher order moments and sample properties differ and these differences increase with the scale $\varepsilon$ of the random part of $A(x)$. The perturbation solution is random and accounts approximately for random fluctuations of the conductivity field. The continuum mechanics solution $u_{0}(x)$ is consistent, i.e., $u_{0}(x)=E[U(x)], 0<x<l$, but does not depend on material properties, in contrast to the perturbation solution that captures these properties approximately.

Figure 5 shows histograms of the difference $\max _{x \in D}\left\{\left|U(x)-U_{\text {pert }}(x)\right|\right\}$ between the exact and perturbation solutions (left panel) and between the exact and continuum mechanics solutions $\max _{x \in D}\left\{\left|U(x)-u_{0}(x)\right|\right\}$ (right panel) for $\varepsilon=0.5$. Since the histogram of $\max _{x \in D}\left\{\left|U(x)-u_{0}(x)\right|\right\}$ is much wider than that of $\max _{x \in D}\left\{\left|U(x)-U_{\text {pert }}(x)\right|\right\}$, we conclude that the perturbation solution provides an improvement of the continuum solution. 


\section{Applications}

The relationship between the continuum mechanics and micromechanics solutions is further explored by solving numerically two stochastic mechanic problems. The first is a beam with random stiffness. It is shown that the variability of the microscale solution $U(x)$ about the continuum mechanics solution $u_{0}(x)$ depends strongly on the correlation structure of the stiffness random field. The second is the transport problem in Eq. 2. Theoretical results related to the convergence of small scale solutions to continuum solutions are developed for one-dimensional problems. Numerical results are presented for two-dimensional transport equations for two quantities of interest, the solutions $u_{0}(x)$ and $U(x)$ and the apparent conductivity for specimens of finite size. These problems further illustrate features and limitations of the continuum mechanics solutions.

We continue to assume that (1) material microstructures are described completely by random fields $A(x), x \in D$, with known probability law, (2) material responses at small scale are actual responses, (3) material properties at large scale used in continuum mechanics are deterministic and can be derived from microscale properties, and (4) continuum mechanics solutions are deterministic functions that constitute approximate material responses.

\subsection{Beam with random stiffness}

Consider a cantilever of length $l>0$ that is fixed at the left end and subjected to a unit concentrated force at the right end. The beam stiffness at microscale is characterized by the real-valued ergodic random field $A(x), 0 \leq x \leq l$, with positive and bounded samples and its stiffness at macroscale is a deterministic constant denoted by $A_{\text {eff }}>0$. The beam displacement based on material properties at these scales satisfy the differential equations

$$
\begin{aligned}
\mathcal{D}[U(x)] & :=A(x) \frac{d^{2}}{d x^{2}}[U(x)]=l-x \quad \text { and }, \\
\mathcal{D}_{0}\left[u_{0}(x)\right] & :=A_{\mathrm{eff}} \frac{d^{2}}{d x^{2}}\left[u_{0}(x)\right]=l-x
\end{aligned}
$$

with solutions

$$
\begin{aligned}
U(x) & =l \int_{0}^{x}\left[\int_{0}^{y} \frac{d z}{A(z)}\right] d y-\int_{0}^{x}\left[\int_{0}^{y} \frac{z d z}{A(z)}\right] d y \quad \text { and } \\
u_{0}(x) & =\frac{l x^{2}}{2 A_{\mathrm{eff}}}(1-x /(3 l)) .
\end{aligned}
$$

under the boundary conditions $U(0)=u_{0}(0)=0$ and $U^{\prime}(0)=u_{0}^{\prime}(0)=0$. The operators $\mathcal{D}$ and $\mathcal{D}_{0}$ have the same functional form but different coefficient, the random field $A(x)$ for $\mathcal{D}$ and the deterministic constant $A_{\text {eff }}$ for $\mathcal{D}_{0}$.

The macroscale solution $u_{0}(x)$ is a deterministic third degree polynomial with coefficients depending on the beam length $l$ and the effective stiffness $A_{\text {eff }}$. The microscale solution $U(x)$ is an inhomogeneous real-valued random field whose samples exhibit various spatial dependence depending on the correlation structure of $A(x)$. If the correlation distance $\xi_{c}>0$ of the random field $B(x)=1 / A(x)$ is much larger than the beam length $l$, 
then $U(x) \simeq B(\cdot) l x^{2}(1-x /(3 l)) / 2=l x^{2}(1-x /(3 l)) /(2 A(\cdot))$ resembles the continuum mechanics solution and its uncertainty is similar to that of $B(\cdot)$. If $\xi_{c} \ll l$, the solution $U(x)$ is less uncertain since it is given by integrals with integrands depending the compliance random field $B(x)$. The following property shows that the continuum mechanics solution $u_{0}(x)$ is consistent under mild conditions on the random stiffness field $A(x)$.

Proposition 3. If $A(x)$ is a homogeneous real-valued random field whose samples are in a bounded interval $[a, b], 0<a<b<\infty$, with probability 1 and $B(x)=1 / A(x)$ satisfies the conditions of the Fubini theorem, then $u_{0}(x)$ and $E[U(x)]$ have the same functional form. If, in addition, $A_{\mathrm{eff}}=1 / E[1 / A(x)]$, then $u_{0}(x)=E[U(x)], x \in(0, l)$.

Proof: First note that the random field $B(x)=1 / A(x)$ exists, is homogeneous, and has bounded samples. The expectation of $U(x)$ in Eq. 15 has the form

$$
\begin{aligned}
E[U(x)] & =l \int_{0}^{x}\left(\int_{0}^{y} E\left[\frac{1}{A(z)}\right] d z\right) d y-\int_{0}^{x}\left(\int_{0}^{y} E\left[\frac{1}{A(z)}\right] z d z\right) d y \\
& =E\left[\frac{1}{A(z)}\right]\left(l \int_{0}^{x}\left(\int_{0}^{y} d z\right) d y-\int_{0}^{x}\left(\int_{0}^{y} z d z\right) d y\right)=E\left[\frac{1}{A(z)}\right]\left(l x^{2} / 2-x^{3} / 6\right),
\end{aligned}
$$

which coincides with the functional form of $u_{0}(x)$. If $A_{\mathrm{eff}}=1 / E[1 / A(\cdot)]$, then $u_{0}(x)=$ $E[U(x)], x \in(0, l)$. The interchange of the expectation and integration operations in the above calculations is legal if $B(x)$ satisfies the conditions of the Fubini theorem, i.e., $B(x, \omega)$ is measurable on the product probability space $((0, l) \times \Omega, \mathcal{G} \times \mathcal{F}, \lambda \times P)$ and $\lambda \times P$-integrable, where $\mathcal{G}$ denotes the Borel $\sigma$-field on $(0, l)$ and $\lambda(d x)=d x$ is the Lebesgue measure.

Suppose the beam stiffness is the random field $A(x)=a+(b-a) F_{\operatorname{Beta}(p, q)}^{-1} \circ \Phi(G(x))$, where $0<a<b<\infty, F_{\operatorname{Beta}(p, q)}$ is a standard Beta distribution with shape parameters $p, q>0$, and $G(x)$ is a homogeneous Gaussian field with mean 0, variance 1, and covariance function $E[G(x+\xi) G(x)]=(1+\lambda|\xi|) \exp (-\lambda|\xi|), \lambda>0$. We note that $A(x)$ satisfies the conditions of Property 3 and that the stiffness $A(x)$ fluctuates randomly in space with values in $[a, b]$. The scale of fluctuation of $A(x)$ depends on the value of $\lambda ; A(x)$ is weakly and strongly correlated for large and small $\lambda$. Strongly correlated fields vary slowly in space so that their scales of fluctuations are large.

The histograms of the tip displacement $U(l)$ in Fig. 6 are for $a=0.1, b=20, p=1 / 2$, $q=3, \lambda=1$ (left panel) and $\lambda=20$ (right panel), and are based on 500 independent samples of $U(l)$. The heavy dots are the expectations $E[U(l)]$; they coincide in the two panels since $E[U(x)]$ depends only on $E[1 / A(x)]$ whose calculation involves only the marginal distribution of $A(x)$. However, the marginal distributions of the displacement fields for $\lambda=1$ and $\lambda=20$ differ significantly, as illustrated by the histograms in this figure.

The continuum mechanics solution with $A_{\text {eff }}=1 / E[1 / A(x)]$ is consistent, i.e., $u_{0}(x)=$ $E[U(x)], x \in(0, l)$. However, it provides no information on the variability and the range of $U(x)$, which depend in a complex manner on the spatial correlation of the stiffness random field. For example, estimates of the standard deviations of $U(l)$ are 0.8272 for $\lambda=1$ and 0.5045 for $\lambda=20$, a result that is consistent with our previous comments on the relationship between the uncertainty in $U(x)$ and the correlation length of $B(x)=1 / A(x)$. Estimates of the probability $P\left(U(l)>u_{\mathrm{cr}}\right)$ based on 10000 samples of $U(l)$ are $0.1423 ; 0.0719$ for $\lambda=1 ; 20$ and $u_{\mathrm{cr}}=1.5$ and $0.1033 ; 0.0181$ for $\lambda=1 ; 20$ and $u_{\mathrm{cr}}=2.0$. The probability 

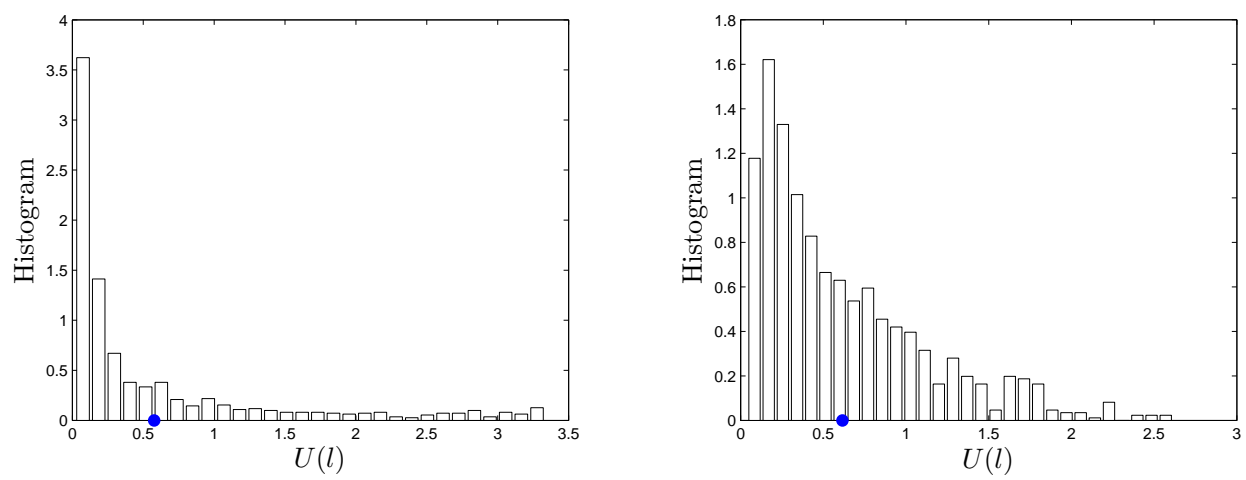

Figure 6: Histograms of $U(l)$ for $\lambda=1$ (left panel) and $\lambda=20$ (right panel)

$P\left(U(l)>u_{\mathrm{cr}}\right)$, which can be interpreted as the beam failure probability, depends strongly on the correlation length of the stiffness random field and the continuum solution cannot capture this dependence. These numerical experiments clearly demonstrate that microscale solutions are required to characterize quantities of interest involving extreme material responses, a common situation in reliability studies.

\subsection{Random conductivity}

Let $U(x), x \in D$, be the solutions of Eq. 2, where $A(x)$ is a real-valued homogeneous random field defined on a probability space $(\Omega, \mathcal{F}, P)$ whose samples are in a bounded interval of $(0, \infty)$, i.e., the events $\left\{\inf _{x \in D}\{A(x)\}>0\right\}$ and $\left\{\sup _{x \in D}\{A(x)\}<\infty\right\}$ have unit probability. Since the coefficients of Eq. 2 are random, this equation is stochastic and its solution $U(x)$ is a random field which is inhomogeneous because of the boundary conditions.

The conductivity field $A(x)$ is described by the translation random field

$$
A(x)=a+(b-a) F_{\operatorname{Beta}(p, q)}^{-1}[\Phi(G(x))], \quad x \in D,
$$

where $F_{\operatorname{Beta}(p, q)}$ denotes the standard Beta distribution with shape parameters $p$ and $q$ and range $[0,1], \Phi$ is the distribution of the Gaussian variable with mean 0 and variance 1 , and $G(x)$ is a real-valued, homogeneous Gaussian random field defined in $\mathbb{R}^{2}$ with mean 0 , variance 1 , and covariance function

$$
c(\tau)=E[G(x) G(x+\xi)]=\exp \left[-\frac{\xi_{1}^{2}+2 \rho \xi_{1} \xi_{2}+\xi_{2}^{2}}{2}\right], \quad \xi=\left(\xi_{1}, \xi_{2}\right) \in \mathbb{R}^{2},
$$

with $|\rho|<1$. In contrast to the samples of $G(x)$ which take values in the entire real line, the samples of $A(x)$ do not leave a bounded interval $[a, b]$ so that they can be viewed as conductivities of specimens selected at random from a population provided $0<a<b<\infty$.

It is assumed that the random field $A(x)$ defines the specimen conductivity at microscale. As previously, the material response at small scale $U(x)$ is considered to be the actual material response. Two quantities of interest are considered, the material response at small scale $U(x)$ and the apparent conductivity

$$
A_{\text {app }}(D)=\frac{1}{l_{2}} \int_{D} A(x) \frac{\partial U(x)}{\partial x_{1}} d x
$$


which describes the overall conductivity of a specimen occupying a bounded set $D[9]$. The apparent conductivity is a random variable whose properties depend on the conductivity field $A(x)$, the microscale solution $U(x)$, the boundary conditions, and the specimen size.

The continuum mechanics solution $u_{0}(x)$ satisfies Eq. 3 which is derived from Eq. 2 with $A_{\text {eff }}$ in place of $A(x)$ and is viewed as an approximation of $U(x)$. The effective conductivity $A_{\text {eff }}$ is a mathematical object that may or may not exist depending on the properties of $A(x)$. It cannot be obtained experimentally or computationally since its determination requires specimens of unbounded size. However, it is possible to construct approximations of and bounds on $A_{\text {eff }}$ under some assumptions $[3,11,15]$. The simplest but widest bounds on $A_{\text {eff }}$ are $[3,12]$

$$
\frac{1}{E[1 / A(x)]} \leq A_{\mathrm{eff}} \leq E[A(x)]
$$

referred to as Voigt and Reuss bounds [3, 12]. The construction of these bounds involves only the marginal distribution of the conductivity field $A(x)$. Following [12], we approximate $A_{\text {eff }}$ by the average of the apparent conductivity defined by Eq. 18 for a sufficiently large specimen. Under this approximation, the effective conductivity used to set continuum problems can be obtained by averaging samples of the apparent conductivity $A_{\text {app }}(D)$ which depends on the small scale conductivity field and material response $U(x)$. The following numerical illustration focus on the relationship between large and small scale material responses. We note that the continuum solution can be inferred from the microscale solution if it is consistent since, in this case, $u_{0}(x)=E[U(x)], x \in D$.

\subsubsection{One-dimensional specimen}

Suppose $D=(0, l)$ is one-dimensional domain and the conductivity field $A(x)$ is strictly positive almost surely (a.s.), i.e., we have $\inf _{x \in D}\{A(x, \omega)\}>0$ for almost all samples $A(x, \omega)$ of $A(x)$. The small and large scale responses satisfy the equations $d\left(A(x) U^{\prime}(x)\right) / d x=0$ and $d\left(A_{\text {eff }} u_{0}^{\prime}(x)\right) / d x=0$ with the boundary conditions $U(0)=u_{0}(0)=0$ and $U(l)=u_{0}(l)=1$. The small scale solution and the specimen apparent conductivity are

$$
\begin{aligned}
U(x) & =\frac{\int_{0}^{x} d y / A(y)}{\int_{0}^{l} d y / A(y)}, \quad x \in[0, l], \quad \text { and } \\
A_{\text {app }}(l) & =\int_{0}^{l} A(x) U^{\prime}(x) d x=\frac{1}{(1 / l) \int_{0}^{l} d y / A(y)},
\end{aligned}
$$

where the latter equality holds since the flow $A(x) U^{\prime}(x)=c$ through the specimen is constant by the defining equation of $U(x)$ so that $U(x)-U(0)=c \int_{0}^{x} d y / A(y)$, which gives the flow $c=1 / \int_{0}^{l} d y / A(y)$ for $x=l$ by using the boundary conditions. The continuum mechanics solution is $u_{0}(x)=x / l, 0<x<l$.

Suppose the specimen conductivity and its inverse are homogeneous random fields with finite variance, i.e., are members of the Hilbert space $L^{2}(\Omega, \mathcal{F}, P)$. The following properties show that the microscale solution $U(x)$ approaches the macroscale solution $u_{0}(x)$ as the uncertainty in $A(x)$ decreases, give limits of $A_{\text {app }}(l)$ as the specimen size increases indefinitely, and determine the effective conductivity. 
Property 4. If $\sup _{x \in D}|A(x, \omega)-\mu| \rightarrow 0, \mu \in \mathbb{R}$, for almost all samples of $A(x)$, i.e., the samples of this field are space-invariant and equal to $\mu$, then $U(x) \rightarrow u_{0}(x)=x / l$ a.s.

Proof: The stochastic integrals in the expression of $U(x)$ can be defined sample-by-sample, i.e., $U(x, \omega)=\int_{0}^{x} d y / A(y, \omega) / \int_{0}^{l} d y / A(y, \omega)$ for almost all $\omega \in \Omega$, so that $U(x, \omega) \rightarrow$ $\int_{0}^{x} d y / \mu / \int_{0}^{l} d y / \mu=x / l=u_{0}(x)$.

Property 5. (i) If $1 / A(x)$ is an ergodic random field, then $\lim _{l \rightarrow \infty} A_{\text {app }}(l)=1 / E[1 / A(\cdot)]$ a.s.; (ii) If the random field $1 / A(x), x \in(0, l)$, is in $L^{2}(\Omega, \mathcal{F}, P)$ and is measurable in $(x, \omega)$, then $A_{\text {app }}(l)$ converges in m.s. to a limit in $L^{2}(\Omega, \mathcal{F}, P)$ denoted by $A_{\text {eff }}$ as $l \rightarrow \infty$; and (iii) Let $\bar{B}(l)=(1 / l) \int_{0}^{l} B(x) d x$ be the spatial average of $B(\cdot)$ over the specimen length, so that $A_{\text {app }}(l)=1 / \bar{B}(l)$. If $\operatorname{Var}[\bar{B}(l)] \rightarrow 0$ as $l \rightarrow \infty$, then $\lim _{l \rightarrow \infty} \bar{B}(l)=E[B(\cdot)]$. The effective conductivity $A_{\text {eff }}$ can be approximated by $1 / \bar{B}(l)$ for $l$ sufficiently large provided $\inf _{0 \leq x \leq l}\{B(x)\}>\alpha>0$ a.s.

Proof: (i) We have $\lim _{l \rightarrow \infty} A_{\text {app }}(l)(\omega)=1 / \lim _{l \rightarrow \infty}(1 / l) \int_{0}^{l} d y / A(y, \omega)=1 / E[1 / A(\cdot)]$ a.s. by the postulated properties of the conductivity field; (ii) Set $X_{n}=(1 /(n \Delta l)) \int_{0}^{n \Delta l}(1 / A(y)) d y$ with $n=1,2, \ldots$ and $\Delta l>0$ and note that $\left\{X_{n}\right\}$ is a Cauchy sequence in the mean square sense, i.e., $E\left[\left(X_{n}-X_{m}\right)^{2}\right] \rightarrow 0$ as $m, n \rightarrow \infty$, since $X_{n}-X_{m}=(m / n-1) X_{m}+$ $(1 /(n \Delta l)) \int_{m \Delta l}^{n \Delta l}(1 / A(y)) d y, n>m ;$

$$
\begin{aligned}
E\left[\left(X_{n}-X_{m}\right)^{2}\right] & =(m / n-1)^{2} E\left[X_{m}^{2}\right]+\frac{1}{(n \Delta l)^{2}} E\left[\left(\int_{m \Delta l}^{n \Delta l}(1 / A(y)) d y\right)^{2}\right] \\
& +2(m / n-1) \frac{1}{n \Delta l} E\left[X_{m} \int_{m \Delta l}^{n \Delta l}(1 / A(y)) d y\right]
\end{aligned}
$$

and the expectations in the expression of $E\left[\left(X_{n}-X_{m}\right)^{2}\right]$ are finite. For example, we have

$$
E\left[X_{m}^{2}\right]=\frac{1}{(m \Delta l)^{2}} \int_{[0, m \Delta l]^{2}} E\left[\frac{1}{A(x) A(y)}\right] d x d y \leq \frac{1}{(m \Delta l)^{2}} \int_{[0, m \Delta l]^{2}} E\left[\frac{1}{A(\cdot)^{2}}\right] d x d y
$$

by Fubini's theorem and Cauchy-Schwarz's inequality, so that $E\left[X_{m}^{2}\right] \leq E\left[1 / A(\cdot)^{2}\right]<\infty$. Similar arguments can be used for the other expectations. This implies that $\left\{1 / X_{n}\right\}$ is Cauchy in $L^{2}$, i.e., $E\left[\left(1 / X_{n}-1 / X_{m}\right)^{2}\right] \rightarrow 0$ as $m, n \rightarrow \infty$, so that it has a limit in $L^{2}$ denoted by $A_{\text {eff }}$; and (iii) The first two moments of $\bar{B}(l)$ are $E[\bar{B}(l)]=E[B(\cdot)]=\mu$ and $E\left[\bar{B}(l)^{2}\right]=(1 / l) \int_{-l}^{l}(1-|v| / l) r(v) d v$. If $\lim _{l \rightarrow \infty} \operatorname{Var}[\bar{B}(l)]=0$ as assumed, the spatial average $\bar{B}(l)$ converges in m.s. to $\mu$. It also converges in probability since, for any $\varepsilon>0$, we have $P(|\bar{B}(l)-\mu|>\varepsilon) \leq \operatorname{Var}[\bar{B}(l)] / \varepsilon^{2}$ by Chebyshev's inequality. The m.s. convergence $1 / \bar{B}(l) \rightarrow 1 / \mu$ follows from

$$
E\left[(1 / \bar{B}(l)-1 / \mu)^{2}\right]=E\left[(\bar{B}(l)-\mu)^{2} /(\mu \bar{B}(l))^{2}\right] \leq E\left[(\bar{B}(l)-\mu)^{2}\right] / \alpha^{2} \rightarrow 0, \quad l \rightarrow \infty,
$$

where the inequality holds since $\inf _{0 \leq x \leq l}\{B(x)\}>\alpha>0$ by assumption.

Property 6. If $A_{\text {app }}(l)$ converges to $A_{\text {eff }}$, the effective conductivity is such that $A_{\text {eff }}=$ $1 / E[1 / A(x)] \leq E[A(x)]$. Moreover, $A_{\text {eff }}$ takes values in $(a, b), 0<a<b<\infty$, if almost all samples of the conductivity field are in this interval. 
Proof: That $A_{\mathrm{eff}}=1 / E[1 / A(\cdot)]$ coincides with the Reuss bound results from the previous property. The following probabilistic arguments show that $A_{\text {eff }}$ is smaller than the Voigt bound $E[A(\cdot)]$. We have

$$
1=\left(E\left[A(x)^{1 / 2} A(x)^{-1 / 2}\right]\right)^{2} \leq E[A(x)] E[1 / A(x)],
$$

which implies $1 / E[1 / A(x)] \leq E[A(x)]$. The second statement follows from the expression of $A_{\mathrm{app}}(l)$ in Eq. 20 .

Numerical results have been obtained for the one-dimensional version of the conductivity field $A(x)$ in Eq. 16 with $a=1, b=20, p=1 / 2, q=3$, and a homogeneous Gaussian field $G(x)$ with mean 0 and correlation function $r_{G}(\xi)=E[G(x+\xi) G(x)]=\exp (-\lambda|\xi|)(1+\lambda|\xi|)$ for $\lambda=10$. Estimates of the mean apparent conductivity $E\left[A_{\mathrm{app}}(l)\right]$ obtained from 500 independent samples of $A_{\text {app }}(l)$ are 2.5859, 2.2045, 2.1332, and 2.1229 for $l=1,10,50$, and 500. Corresponding estimates of the standard deviation of the apparent conductivity are $\operatorname{Std}\left[A_{\text {app }}(l)\right]=1.2691,0.2806,0.1203$, and 0.0426. The effective conductivity is $A_{\text {eff }}=$ $1 / E[1 / A(x)]=2.0823$.

These results are in agreement with our theoretical arguments specifying conditions under which $A_{\text {eff }}$ can be approximated by $A_{\text {app }}(l)$ for a sufficiently large $l$. Since the correlation function has the property $\lim _{\xi \rightarrow \infty} r_{G}(\xi)=E[G(x+\xi) G(x)]=0, G(x)$ is ergodic ([1], Theorem 6.5.4) and so are the random fields $A(x)$ and $1 / A(x)$ as measurable mappings of this Gaussian field. The above numerical results show that the uncertainty in $A_{\text {app }}(l)$ decreases with the specimen size $l$ so that $E\left[A_{\text {app }}(l)\right]$ provides a robust approximation of the effective conductivity for sufficiently large specimens such that $\operatorname{Std}\left[A_{\text {app }}(l)\right]$ is small.

We conclude with the observation that the microscale solution in Eq. 20 can be given in the form $U(x)=A_{\text {app }}(l)(1 / l) \int_{0}^{x} d y / A(y)$ so that, for a sufficiently large $l$, can be approximated by $U(x) \simeq A_{\text {eff }}(1 / l) \int_{0}^{x} d y / A(y)$. The expectation of this approximate expression of $U(x)$ is $E[U(x)] \simeq A_{\text {eff }} E[1 / A(\cdot)] \simeq x / l$ for a sufficiently large $x$, which coincides with the continuum mechanics solution. Accordingly, the continuum solution is consistent under the stated assumptions.

\subsubsection{Two-dimensional specimen}

We consider the same quantities of interest, the microscale solution $U(x)$ and the apparent conductivity $A_{\text {app }}(D)$ in Eq. 18. The continuum mechanics solution $u_{0}(x)$ is defined by Eq 3, i.e., Eq 2 with $A_{\text {eff }}$ in place of $A(x)$, and has the expression $u_{0}(x)=x_{1} / l_{1}$. As for the one-dimensional specimens, the continuum solution does not depend on material properties.

To assess the performance of the continuum formulation, we examine the relationships between the expectations of the microscale solution $U(x)$ and the continuum mechanics solution $u_{0}(x)$, compare histograms of $A_{\text {app }}(D)$ with possible ranges of $A_{\text {eff }}$, and calculate mean square errors between $A_{\text {app }}(D)$ and possible values of $A_{\text {eff }}$. The following numerical results are for $l_{1}=20, l_{2}=10, a=1$ and $a=0.1, b=20, p=1 / 2, q=3$, and $\rho=0.7$. The plots in Figs. 7 to 9 are for $a=1$ and those in Figs. 10 to 12 are for $a=0.1$. Samples of $U(x)$ are obtained from samples of the conductivity field $A(x)$ and corresponding solutions of Eq. 2 obtained by existing deterministic solvers. Samples of the apparent conductivity $A_{\text {app }}(D)$ are calculated from Eq. 18 and samples of $A(x)$ and $U(x)$.

The left and right panels in Fig. 7 show estimates of the mean and standard deviation of 

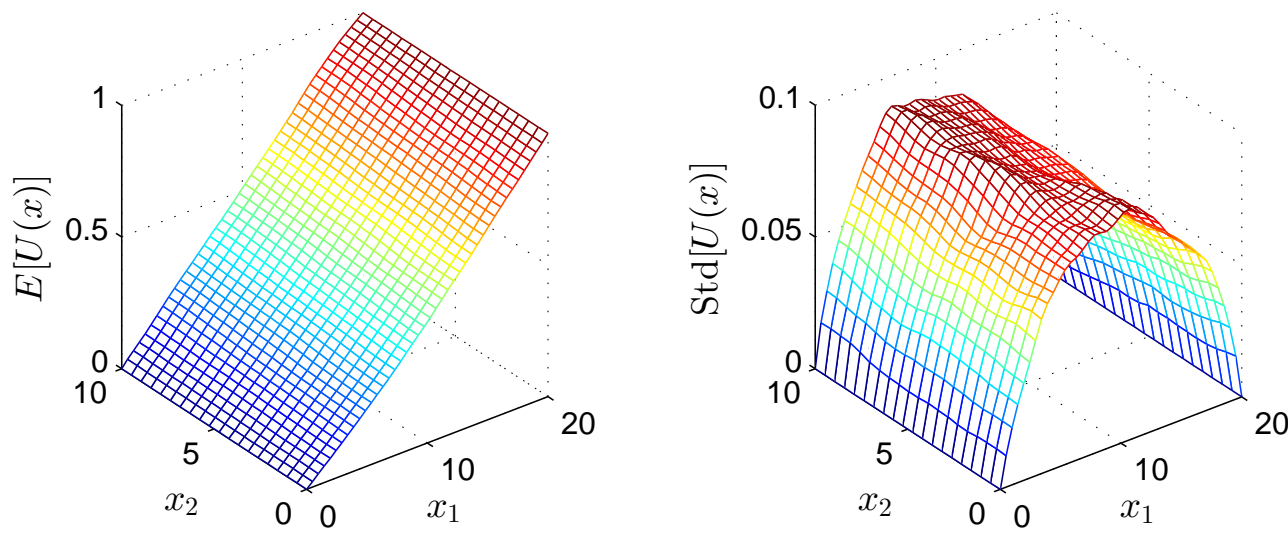

Figure 7: Estimates of $E[U(x)]$ (left panel) and $\operatorname{Std}[U(x)]$ (right panel) for $a=1$ and $b=20$

$U(x)$ obtained from 100 independent samples of $U(x)$. The continuum solution $u_{0}(x)=x_{1} / l_{1}$ is consistent since it is approximately equal to the estimate of the expectation $E[U(x)]$ of the microscale solution $U(x)$ in this figure. However, there are notable differences between $u_{0}(x)$ and $U(x)$ as quantified in the right panel of Fig. 7 that shows the standard $\operatorname{Std}[U(x)]$ deviation of $U(x)$.

Differences between $u_{0}(x)$ and $U(x)$ are further quantified by the histograms of the microscale solution $U\left(l_{1} / 2, l_{2} / 2\right)$ and the apparent conductivity $A_{\text {app }}(D)$. The histograms of these random variables are shown in the left and right panels of Fig. 8 and are based on based on 100 independent samples of $A(x)$ and the corresponding samples of $U(x)$ and $A_{\text {app }}(D)$.
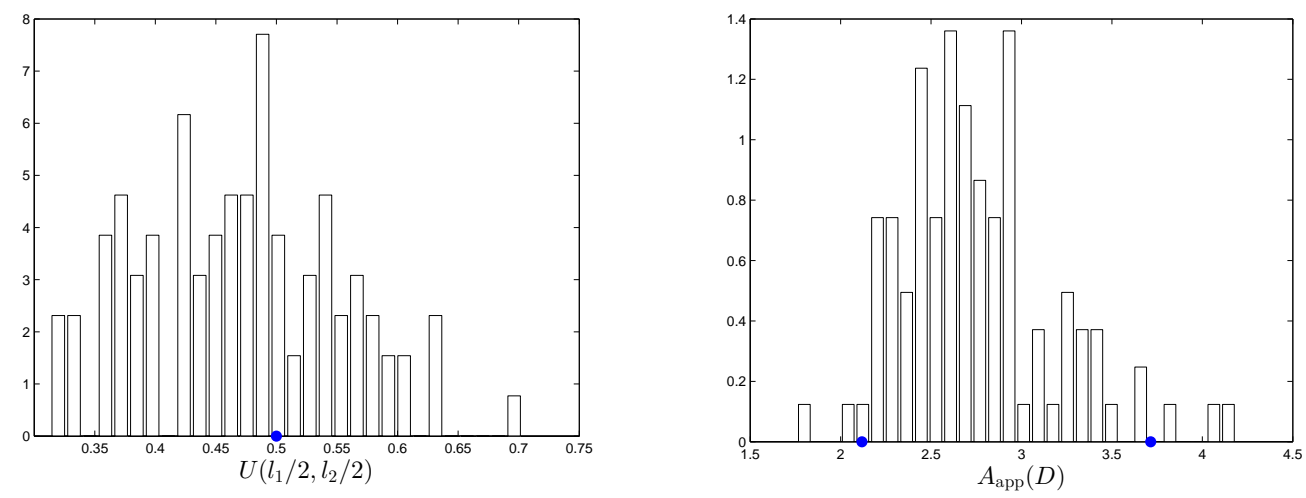

Figure 8: Histograms of $U\left(l_{1} / 2, l_{2} / 2\right)$ (left panel) and $A_{\text {app }}(D)$ (right panel) for $a=1$ and $b=20$

The heavy dot in the left panel is the continuum solution $u_{0}\left(l_{1} / 2, l_{2} / 2\right)=0.5$. The heavy dots in the right panel mark the range $(1 / E[1 / A(x)], E[A(x)])=(2.1176,3.7143)$ of $A_{\text {eff }}$ that corresponds to the Voigt and Reuss bounds. It includes the expectation $E\left[A_{\text {app }}(D)\right]=2.7513$ of apparent conductivity. The actual values of $A_{\text {eff }}$ is unknown.

Figure 9 shows two samples of $U(x)$ (left panels) and the discrepancies between these samples and the continuum solution $u_{0}(x)$ (right panels). In contrast to the continuum 

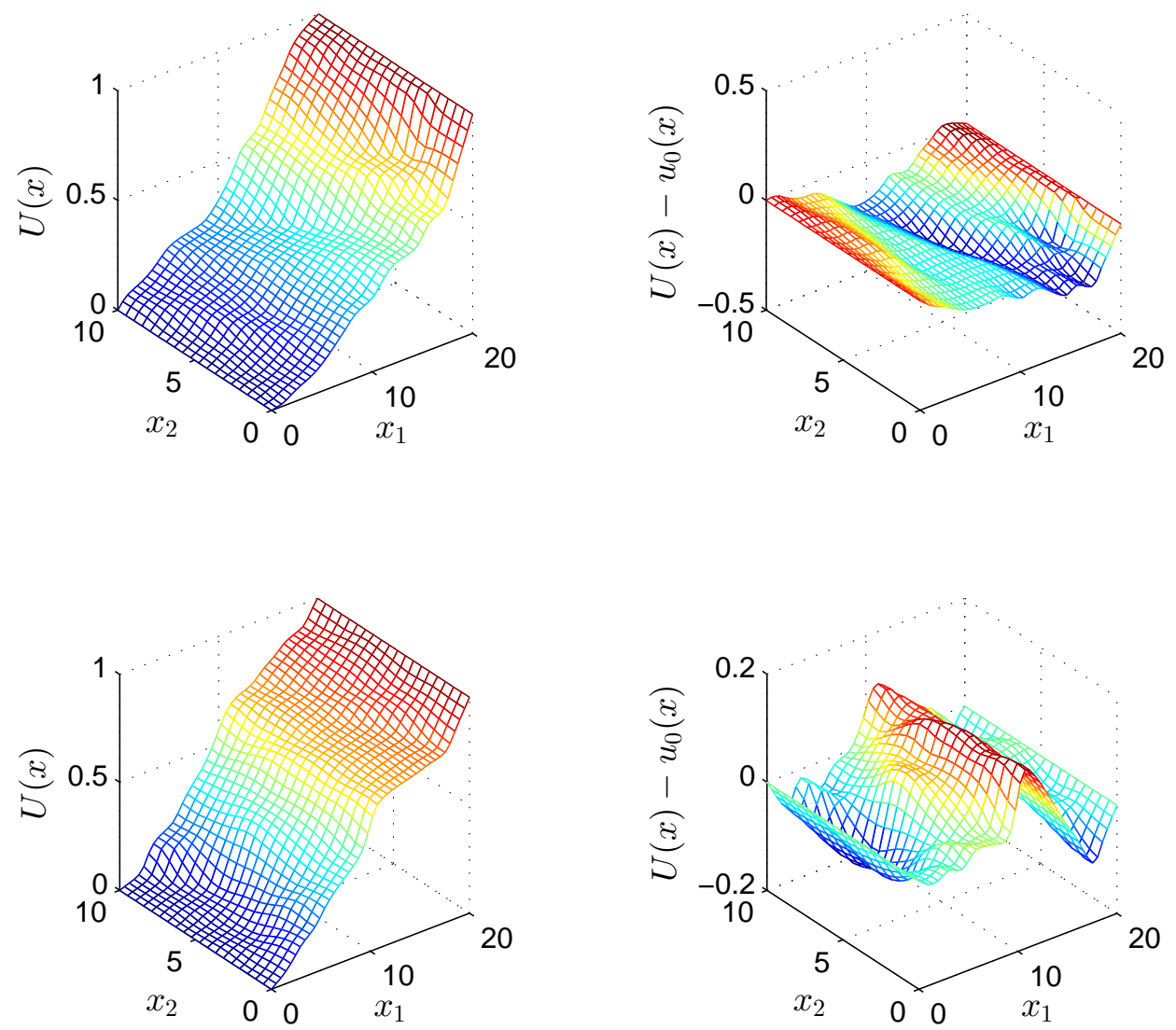

Figure 9: Samples of $U(x)$ (left panels) and corresponding samples of $U(x)-u_{0}(x)$ (right panels) for $a=1$ and $b=20$

mechanics solution, which is one-dimensional and independent of material properties, the microscale solutions vary with both components of $x=\left(x_{1}, x_{2}\right)$ and depend on material properties, as illustrate by the significant sample-to-sample variation of $U(x)$.

Figures 10 to 12 show plots as in Figs. 7 to 9 for $A(x)$ in the range $(a=0.1, b=20)$, rather than $(a=1, b=20)$. Estimates of the mean and standard deviation on $U(x)$ are in the left and right panels of Fig. 10. They are obtained from 100 independent samples of $U(x)$. The continuous solution $u_{0}(x)$ seems to be consistent since it is approximately equal to the estimate of the expectation of $U(x)$ shown in the figure. Histograms of $U\left(l_{1} / 2, l_{2} / 2\right)$ (left panel) and $A_{\text {app }}(D)$ (right panel) are shown in Fig. 11. They are obtained from 100 independent samples of $A(x)$, the corresponding samples of $U(x)$, and Eq. 18. The heavy dots in the figure have the same meaning as in Fig. 8. As previously, the range $(0.5456,2.9429)$ of $A_{\text {eff }}$ contains the expectation $E\left[A_{\text {app }}(D)\right]=1.2453$ of the specimen apparent conductivity. The left and right panels in Fig. 12 show two samples of $U(x)$ and the discrepancy between these samples and $u_{0}(x)$.

The increase of the uncertainty in the conductivity field from the range $(a, b)=(1,20)$ 

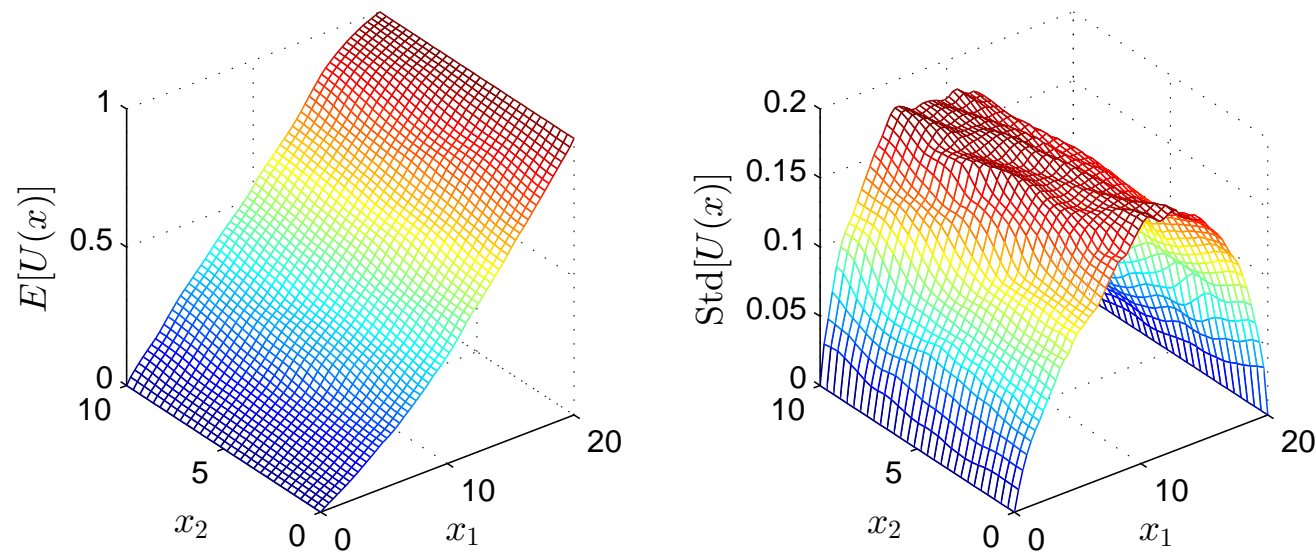

Figure 10: Estimates of $E[U(x)]$ (left panel) and $\operatorname{Std}[U(x)]$ (right panel) for $a=0.1$ and $b=20$
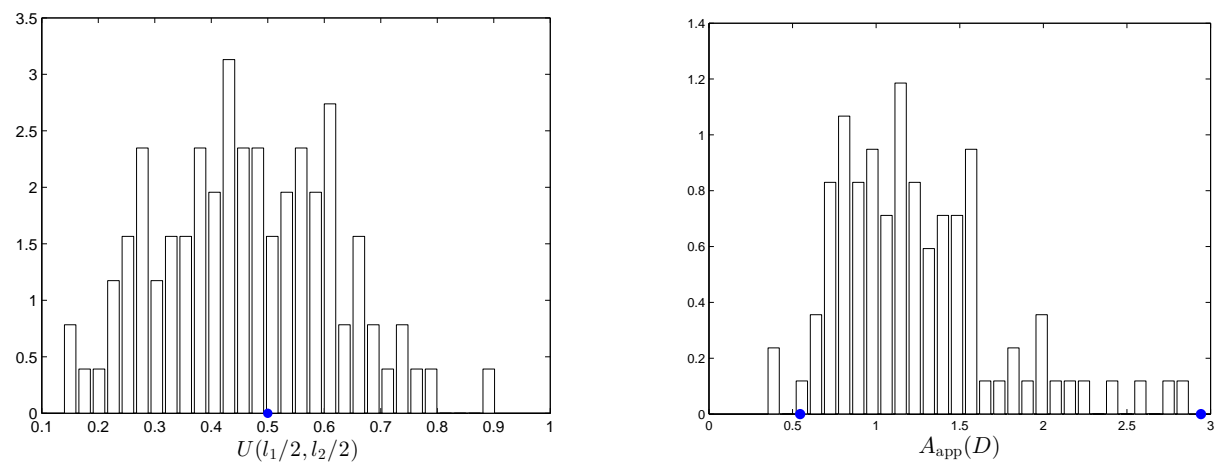

Figure 11: Histograms of $U\left(l_{1} / 2, l_{2} / 2\right)$ (left panel) and $A_{\text {app }}$ (right panel) for $a=0.1$ and $b=20$

to $(a, b)=(0.1,20)$ results in larger differences between the samples of $U(x)$ and the continuum solution $u_{0}(x)$, as illustrated by the standard deviation of $U(x)$ and the samples of $U(x)-u_{0}(x)$. Also, the range of $A_{\text {eff }}$ increases from 3.7143-2.1176=1.5967 for $a=1$ to $2.9429-0.5456=2.3973$ for $a=0.1$. This observation is consistent with findings in [17] which recommend to use microscale solutions for random microstructures with high variance.

Figures 13 and 14 further examine the relationship between the microscale and continuum solutions and suggest a value for the specimen global conductivity that is optimal in some sense. Figure 13 shows 100 samples of $U(x)$ along $x_{2} \in\left[0, l_{2}\right]$ for $x_{1}=l_{1} / 2$ (left panel) and differences $U\left(l_{1} / 2, l_{2} / 2\right)-u_{0}\left(l_{1} / 2, l_{2} / 2\right)$ for 100 samples of $U(x)$ (right panel). While $U\left(l_{1} / 2, x_{2}\right)$ and $U\left(l_{1} / 2, l_{2} / 2\right)$ vary significantly from sample to sample, $u_{0}\left(l_{1} / 2, x_{2}\right)=$ $u_{0}\left(l_{1} / 2, l_{2} / 2\right)=0.5$. The plots clearly illustrate the limited information provided by the continuum solution and raise questions about its use in design.

Figure 14 displays the mean square error $E\left[\left(A_{\text {app }}(D)-A\right)^{2}\right]$ between the apparent conductivity and a constant, deterministic conductivity $A$ assumed to take values in the range of $A_{\text {eff }}$ defined by the Voigt and Reuss bounds. The left and right panels correspond 

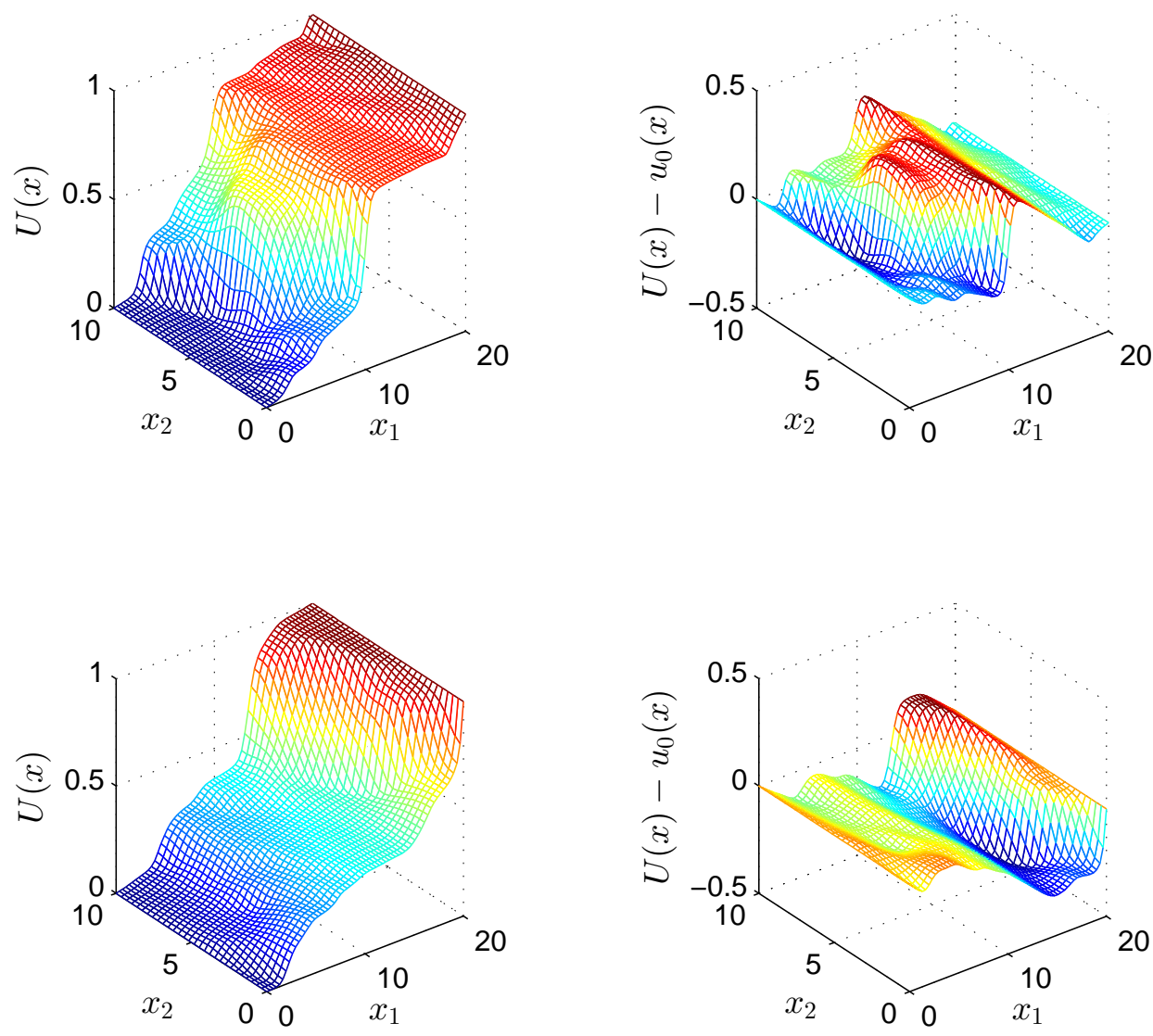

Figure 12: Samples of $U(x)$ (left panels) and corresponding samples of $U(x)-u_{0}(x)$ (right panels) for $a=0.1$ and $b=20$

to $a=1$ and $a=0.1$, respectively. The errors are larger than the variance of $A_{\text {app }}(D)$ because $E\left[\left(A_{\text {app }}(D)-A\right)^{2}\right]=E\left[\left(\left(A_{\text {app }}(D)-E\left[A_{\text {app }}(D)\right]\right)+\left(E\left[A_{\text {app }}(D)\right]-A\right)\right)^{2}\right]=\operatorname{Var}\left[A_{\text {app }}(D)\right]+$ $\left(E\left[A_{\text {app }}(D)\right]-A\right)^{2} \geq \operatorname{Var}\left[A_{\text {app }}(D)\right]$. We note that this error is larger that 0.4 for all values of $A$ in the range considered for the effective conductivity $A_{\text {eff }}$, and that the error reaches its minimum value for $A=E\left[A_{\text {app }}(D)\right]$. This numerical observation is consistent with the estimate $A_{\text {eff }}=E\left[A_{\text {app }}(D)\right]$ proposed in [12] under some conditions.

We summarize the results of the numerical experiments in this section by the following four comments. First, the microscale and macroscale solutions differ qualitatively. The small scale solution is a random field that depends on both spatial coordinates and material properties. In contrast, the continuum solution $u_{0}(x)$ is a deterministic function that is independent of the $x_{2}$-coordinate and material properties. Second, the continuum solution $u_{0}(x)$ is consistent, i.e., $u_{0}(x)=E[U(x)], x \in D$, irrespective of the value of $A_{\text {eff }}$. The effective conductivity $A_{\text {eff }}=E\left[A_{\text {app }}(D)\right]$ has the smallest mean square error relative to the apparent conductivity $A_{\text {app }}(D)$. Third, the differences between the continuum solution $u_{0}(x)$ and samples of the microscale solution $U(x)$ and between the effective conductivity $A_{\text {eff }}$ and 

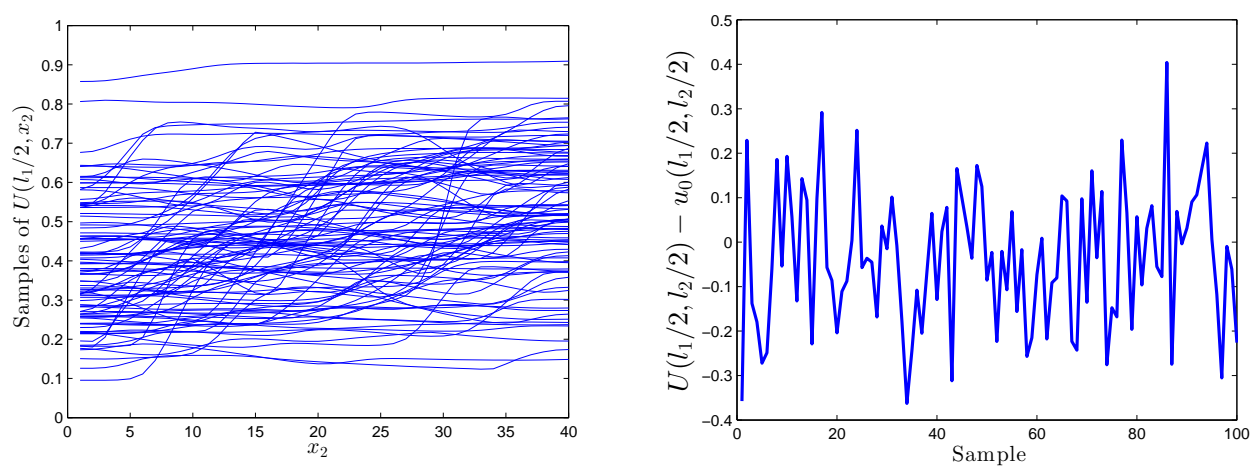

Figure 13: Samples of $U\left(l_{1} / 2, x_{2}\right)$ (left panel) and $U\left(l_{1} / 2, l_{2} / 2\right)-u_{0}\left(l_{1} / 2, l_{2} / 2\right)$ (right panel) for $a=0.1$ and $b=20$
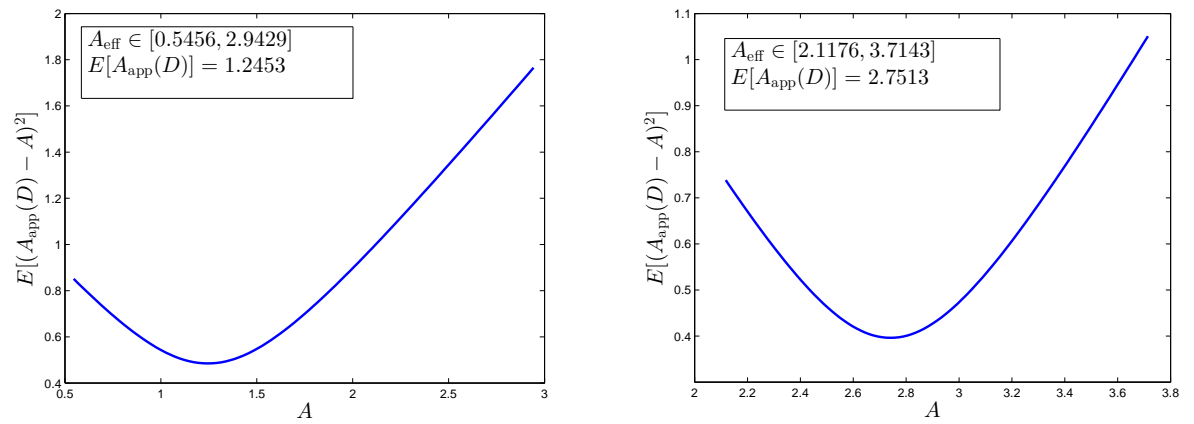

Figure 14: Mean square errors $E\left[\left(A_{\mathrm{app}}(D)-A\right)^{2}\right]$ for $a=1$ (left panel) and $a=0.1$ (right panel)

the apparent conductivity $A_{\mathrm{app}}(D)$ can be significant, and they depend on the uncertainty in the conductivity field, as illustrated by results for conductivities in the ranges $(a, b)=$ $(1,20)$ and $(a, b)=(0.1,20)$. Fourth, the mean square error $E\left[\left(A_{\text {app }}(D)-A\right)^{2}\right]$ between the specimen apparent conductivity $A_{\text {app }}(D)$ and a constant, deterministic conductivity $A$ reaches its minimum at $A=E\left[A_{\mathrm{app}}(D)\right]$.

\section{General comments}

The overall performance of the continuum mechanics solution under the consistency metric is remarkable. The continuum solutions are consistent for the following quantities of interest: (1) the elongation $U(x)$ of a rod with random stiffness considered in Example 1 for $A_{\text {eff }}=1 / E[1 / A(x)],(2)$ the component forces $Q_{i}(\delta)$ of the parallel system with independent components in Example 2 for $K_{\text {eff }}=E\left[K_{1}\right]$, (3) the displacement function of a beam with random stiffness discussed in Sect. 4.1 for $A_{\mathrm{eff}}=1 / E[1 / A(x)]$, (4) the solutions of the stochastic transport equations in Sect. 4.2 irrespective of the value of $A_{\text {eff }}$, and (5) the flow for the stochastic transport equations in Sect. 4.2 for $A_{\text {eff }}=E\left[A_{\text {app }}(D)\right]$, where $\left[A_{\text {app }}(D)\right.$ denotes the apparent conductivity of a specimen in a $D$. 
The continuum solution is not consistent for the quantity of interest $Q_{\max }(\delta)$ calculated in Example 2, an expected result since the maximum component force is a local feature. Consistency is a desirable attribute that only assures that the continuum solution is an unbias estimate of the average microscale solution. However, it is a rather weak requirement that provides no information beyond the mean of the microscale solution. For example, (1) the probability law of the microscale solutions of the rod elongation $U(x)$ in Fig. 1 depends in a complex manner of the spatial correlation of the stiffness random field $A(x)$ but this dependence is not noted by the continuum solution, (2) the histograms of the extreme force $Q \max (\delta)$ in Fig. 2 depend strongly on the distribution of the stiffness $K_{1}$ but this dependence is not capture by the continuum solution, (3) the flow in Fig. 3 predicted by the continuum solution is a single number that gives no indication of the distribution of the apparent conductivity $A_{\mathrm{app}}(D)$ and its dependence on the probability law of the conductivity field $A(x)$, (4) the continuum mechanics gives the same displacement for the beam in Fig. 6 although the microscale solution $U(x)$ changes significantly with the spatial correlation of the stiffness random field $A(x)$, and (5) the continuum solution of the stochastic conductivity equation misses essential features of the response, as illustrated in Figs. 9 and 12.

Since the continuum mechanics solution is at best an estimate of the expectation of the microscale solution, cannot capture the dependence of the microscale solution on the spatial correlation of the stiffness/conductivity random field, and can miss essential features of material response, it cannot be used to design systems with specified reliability levels. Microscale solutions need to be used for this purpose.

\section{Conclusions}

Material properties at small scale vary randomly in space and so are corresponding responses. Random fields have been used to characterize both material properties and responses. Continuum mechanics uses coarser descriptions of material properties, referred to as bulk or effective properties. Since these properties are deterministic so are material responses provided source terms and end conditions are deterministic. We viewed material properties and responses in continuum mechanics as approximations of those in micromechanics. Our premise was that material responses at different scales must agree in some sense. Since microscale and continuum solutions are random and deterministic, it is only possible to compare continuum solutions with statistics of micromechanics solutions.

The overall objective of the paper of assessing differences and similarities between material responses at different scales has been addressed extensively in the literature. Most of the existing studies on this topic focus on the relationships between effective and apparent properties, the development of bounds on effective properties from apparent properties, and the construction of bounds on the discrepancy between material responses at different scales expressed in terms of global metrics, e.g., energy norms. In contrast, this study proposes a new framework for assessing differences and similarities between material responses at different scales based on the theory of stochastic differential equations. In this framework, material responses at different scales are solutions of stochastic/deterministic equations with the same functional form but different coefficients, which depend on the resolution with which material properties are represented. 
The proposed approach has some notable features. First, bounds on differences between material responses at different scales quantified by various metrics result from the theory of stochastic equations. The metrics are not restricted to energy norms or other global metrics, which cannot capture local response features. Second, the bounds are for differences between material responses at arbitrary scales and relate simply to differences between material properties at these scales. They are not derived from bounds on material properties as in most current studies, e.g., bounds on effective material properties. Third, recent advances on practical methods for solving stochastic equations provide efficient solutions for complex problems of the type encountered in micromechanics.

Theoretical considerations and numerical examples have been used to support and illustrate the proposed approach of assessing differences between responses at different scales via concepts of stochastic differential equations. The construction of bounds on differences between material responses at different scales has been discussed in Sects. 3.1 and 3.2 for stochastic transport equations with conductivities of arbitrary and small uncertainty. It was found in a numerical illustration that the first order perturbation solution is more accurate than the continuum solution. Numerical examples involving rods with random stiffnesses in tension, parallel systems with random properties, and one- and two-dimensional stochastic transport problems have shown that continuum solutions may or may not be consistent and may miss essential features of material responses at small scale depending on the problem and quantity of interest. It was also found that the mean square error between a specimen apparent conductivity and the material effective conductivity is minimized by setting the effective conductivity equal to the expectation of the apparent conductivity.

\section{Acknowledgements}

The work reported in this paper has been supported by the National Science Foundation under grand CMMI-0969150. This support is gratefully acknowledged.

\section{References}

[1] R. J. Adler. The Geometry of Random Fields. John Wiley \& Sons, New York, 1981.

[2] K. Alzebdeh and M. Ostoja-Starzewski. Micromechanically based stochastic finite elements: length scales and anisotropy. Probabilistic Engineering Mechanics, 11:205-214, 1996.

[3] M. J. Beran. Statistical Continuum theories. Interscience Publishers, New York, 1968.

[4] M. Chen, Z. Chen, and G. Chen. Approximate Solutions of Operator Equations. World Scientific, NewYork, 1997.

[5] L. L. Graham-Brady, E. F. Siragy, and S. C. Baxter. Analysis of heterogeneous composites based on moving-window techniques. Journal of Engineering Mechanics, ASCE, 129:1054-1064, 2003. 
[6] M. Grigoriu. A nearest neighbor probabilistic model for aluminum polycrystals. Journal of Engineering Mechanics, 136(7):821-829, 2010. doi: 10.1061/(ASCE)EM.19437889.0000163.

[7] M. Grigoriu. Stochastic Systems. Uncertainty Quantification and Propagation. Springer Series in Reliability Engineering. Springer, London Heidelberg New York Dordrecht, 2012. ISBN 978-1-4471-2326-9, ISBN 978-1-4471-2327-9 (eBook).

[8] M. Grigoriu. Response statistics for random heterogeneous microstructures. SIAM, Journal of Uncertainty Quantification, 2(1), 2014.

[9] M. Grigoriu and K. D. Papoulia. Effective conductivity by a probability-based local method. Journal of Applied Physics, 98:033706(1-10), 2005.

[10] S. Hazanov and C. Huet. Order relationships for boundary conditions effects in heterogeneous bodies smaller than the representative volume. Journal of the Mechanics and Physics of Solids, 1995.

[11] E. J. Hinch. Perturbation Methods. Cambridge University Press, Cambridge, 1994.

[12] C. Huet. Applications of variational concepts to size effects in elatic heterogeneous bodies. Journal of the Mechanics and Physics of Solids, 38(6):813-841, 1990.

[13] T. Kanit, S. Forest, T. Galliet, V. Mounoury, and D. Jeulin. Determination of the size of the representative volume element for random composites: statistical and numerical approach. International Journal of Solids and Structures, 40:3647-3679, 2003.

[14] M. Ostoja-Starzewski. Micromechanics as a basis of continuum random fields. Applied Mechanics Reviews, 47:221-230, 1994.

[15] M. Ostoja-Starzewski. Microstrucutral disorder, mesoscale finite elements and microscopic response. Proceedings of the Royal Society London, 455:3189-3199, 1999.

[16] M. Ostoja-Starzewski. Microstructural Randomness and Scaling in Mechanics of Materials. Chapman \& Hall/CRC, New York, 2007.

[17] M. Ostoja-Starzewski and X. Wang. Stochastic finite elements as a bridge between random material microstructure and global response. Campater Methsds in Applied Mechanics and Engineering, 168:35-49, 1999.

[18] B. D. Reddy. Introduction to Functional Analysis with Applications to Boundary Value Problems and Finite Elements. Springer-Verlag, New York, 1998.

[19] S. I. Resnick. A Probability Path. Birkhäuser, Boston, 1998. 

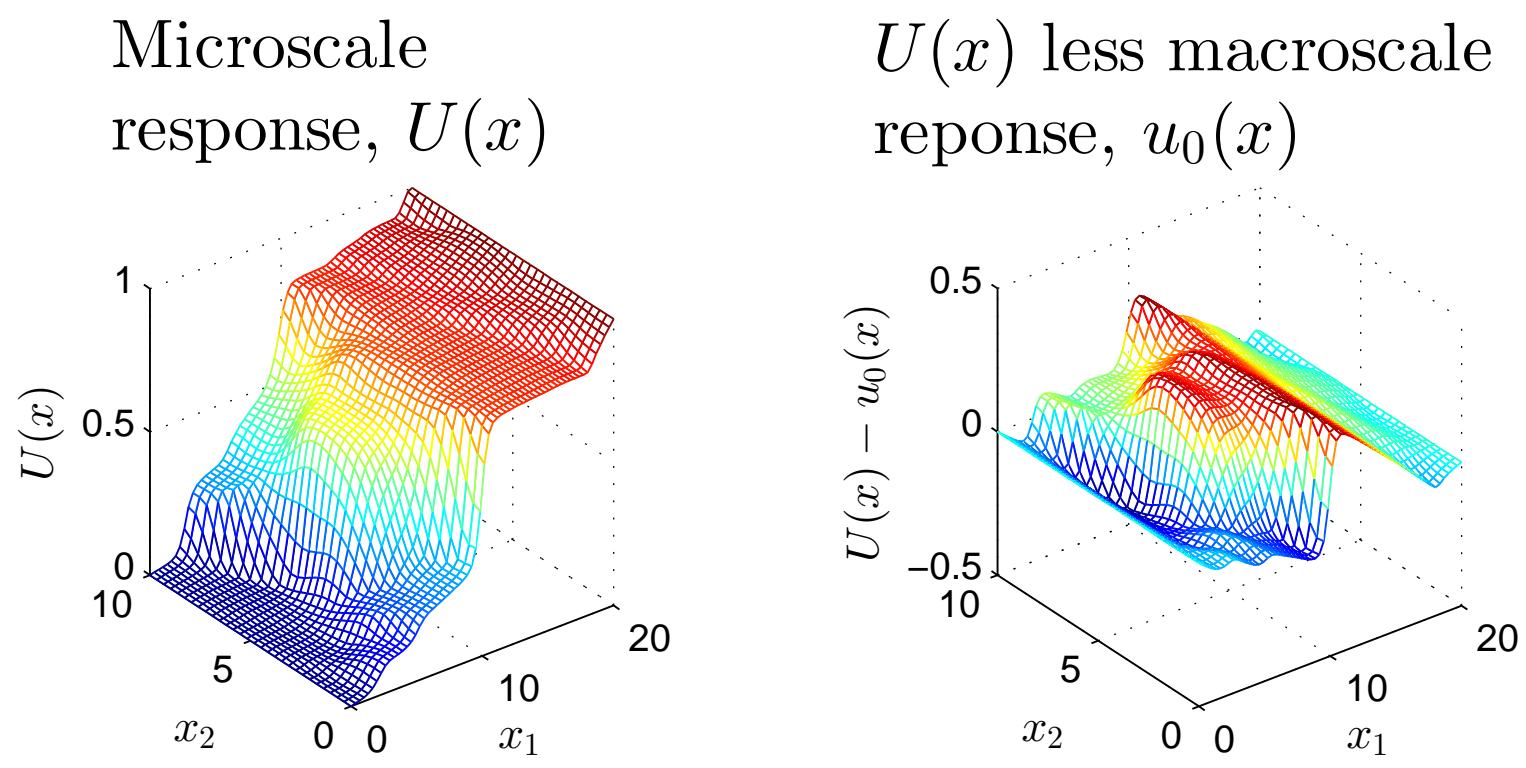